\title{
Electroweak and QCD corrections to $Z$ and $W$ pole observables in the standard model EFT
}

\author{
Sally Dawson $\oplus^{1}$ and Pier Paolo Giardino $\oplus^{2}$ \\ ${ }^{1}$ Department of Physics, Brookhaven National Laboratory, Upton, New York 11973, USA \\ ${ }^{2}$ Instituto de Fisica Teorica, UAM-CSIC, Cantoblanco, 28049 Madrid, Spain
}

(Received 9 September 2019; published 7 January 2020)

\begin{abstract}
We compute the next-to-leading order QCD and electroweak corrections to $Z$ and $W$ pole observables using the dimension-6 Standard Model effective field theory (SMEFT) and present numerical results that can easily be included in global fitting programs. Limits on SMEFT coefficient functions are presented at leading order and at next-to-leading order under several assumptions.
\end{abstract}

DOI: 10.1103/PhysRevD.101.013001

\section{INTRODUCTION}

The LHC experiments provide strong evidence that the $S U(3) \times S U(2) \times U(1) \quad$ Standard Model $(\mathrm{SM})$ gauge theory describes physics at the electroweak scale [1]. To date, there is no evidence of new interactions or high mass particles. Taken together, these features suggest that the weak scale can be described by an effective field theory (SMEFT) having the SM as its low energy limit. The SMEFT is defined by an infinite tower of dimension- 6 and higher operators, involving only the SM particles and assumes that the Higgs boson is part of an $S U(2)$ doublet [2]. The effects of the higher dimension operators are suppressed by powers of a high scale, $\Lambda$, and we assume that the most numerically relevant operators are those of dimension-6. All possible new physics phenomena are contained in the coefficient functions.

Numerous studies have been performed extracting limits on the coefficients of dimension- 6 operators from global fits to Higgs measurements, vector boson pair production, electroweak measurements at the $Z$ and $W$ poles, top quark measurements, and low energy data [3-10]. Typically, these fits use the most accurately known SM predictions, while the SMEFT effects are treated at lowest order (LO). A program of calculations has begun to treat the SMEFT contributions at next-to-leading order (NLO), for both the QCD and electroweak (EW) contributions. The SMEFT QCD corrections to gauge boson pair production $[11,12]$ and top quark production and decay [13-16] are known. The electroweak SMEFT corrections to Higgs decays to $b \bar{b}$ [17-19], $\gamma \gamma$ [20-23], $Z \gamma$ [24,25], $Z Z$ [24], and $W W$ [20]

Published by the American Physical Society under the terms of the Creative Commons Attribution 4.0 International license. Further distribution of this work must maintain attribution to the author(s) and the published article's title, journal citation, and DOI. Funded by SCOAP . have also been computed, along with partial corrections to the Drell Yan process [26]. The SMEFT NLO corrections to $Z$ pole decays at NLO are also only partially known [27-29].

In this work, we take a major step by computing the NLO EW and QCD corrections in the SMEFT to $Z$ and $W$ pole observables. We assume flavor universality and use the Warsaw basis [30,31]. We are particularly interested in the numerical effects of the NLO corrections on the global fits. In Sec. II, we review the basics of the SMEFT theory and in Sec. III, we describe our NLO calculations. Our results are given in Sec. IV and Appendix, which contains numerical expressions for the $Z$ and $W$ pole observables, as well as limits on the SMEFT coefficients at LO and NLO. Section V contains some conclusions and a discussion of the implications of our results for global fits.

\section{SMEFT BASICS}

The SMEFT parametrizes new physics through an expansion in higher dimensional operators,

$$
\mathcal{L}=\mathcal{L}_{\mathrm{SM}}+\Sigma_{k=5}^{\infty} \Sigma_{i=1}^{n} \frac{\mathcal{C}_{i}^{k}}{\Lambda^{k-4}} O_{i}^{k},
$$

where the $S U(3) \times S U(2)_{L} \times U(1)_{Y}$ invariant dimension- $k$ operators are constructed from SM fields and all of the effects of the beyond the SM (BSM) physics reside in the coefficient functions, $\mathcal{C}_{i}^{k}$. We assume all coefficients are real and do not consider the effects of $C P$ violation. We use the Warsaw basis $[30,31]$ and at tree level (neglecting flavor) there are 10 dimension- 6 operators contributing to the $Z$ and $W$ pole observables of our study. These operators are listed in Table I, where $\phi$ is the $S U(2)_{L}$ doublet, $\tau^{a}$ are the Pauli matrices, $D_{\mu}=\partial_{\mu}+i g_{s} T^{A} G_{\mu}^{A}+i g_{2} \frac{\tau^{a}}{2} W_{\mu}^{a}+i g_{1} Y B_{\mu}, q^{T}=\left(u_{L}, d_{L}\right)$, $l^{T}=\left(\nu_{L}, e_{L}\right), \quad W_{\mu \nu}^{a}=\partial_{\mu} W_{\nu}^{a}-\partial_{\nu} W_{\mu}^{a}-g_{2} \epsilon^{a b c} W_{\mu}^{b} W_{\nu}^{c}$, $\phi^{\dagger} i \stackrel{\leftrightarrow}{D_{\mu}} \phi=i \phi^{\dagger}\left(D_{\mu} \phi\right)-i\left(D_{\mu} \phi\right)^{\dagger} \phi, \quad$ and $\quad \phi^{\dagger} i \stackrel{\leftrightarrow}{D_{\mu}^{a}} \phi=$ $i \phi^{\dagger} \tau^{a} D_{\mu} \phi-i\left(D_{\mu} \phi\right)^{\dagger} \tau^{a} \phi$. 
TABLE I. Dimension-6 operators contributing to the $Z$ and $W$ pole observables of this study at tree level.

\begin{tabular}{|c|c|c|c|c|c|}
\hline $\mathcal{O}_{l l}$ & $\left(\bar{l} \gamma_{\mu} l\right)\left(\bar{l}^{\mu} l\right)$ & $\mathcal{O}_{\phi W B}$ & $\left(\phi^{\dagger} \tau^{a} \phi\right) W_{\mu \nu}^{a} B^{\mu \nu}$ & $\mathcal{O}_{\phi D}$ & $\left(\phi^{\dagger} D^{\mu} \phi\right)^{*}\left(\phi^{\dagger} D_{\mu} \phi\right)$ \\
\hline $\mathcal{O}_{\phi e}$ & $\left(\phi^{\dagger} i \stackrel{\leftrightarrow}{D}_{\mu} \phi\right)\left(\bar{e}_{R} \gamma^{\mu} e_{R}\right)$ & $\mathcal{O}_{\phi u}$ & $\left(\phi^{\dagger} i \stackrel{\leftrightarrow}{D}_{\mu} \phi\right)\left(\bar{u}_{R} \gamma^{\mu} u_{R}\right)$ & $\mathcal{O}_{\phi d}$ & $\left(\phi^{\dagger} i \stackrel{\leftrightarrow}{D_{\mu}} \phi\right)\left(\bar{d}_{R} \gamma^{\mu} d_{R}\right)$ \\
\hline $\mathcal{O}_{\phi q}^{(3)}$ & $\left(\phi^{\dagger} i \stackrel{\leftrightarrow}{D}_{\mu}^{a} \phi\right)\left(\bar{q} \tau^{a} \gamma^{\mu} q\right)$ & $\mathcal{O}_{\phi q}^{(1)}$ & $\left(\phi^{\dagger} i \stackrel{\leftrightarrow}{D}_{\mu} \phi\right)\left(\bar{q} \tau^{a} \gamma^{\mu} q\right)$ & $\mathcal{O}_{\phi l}^{(3)}$ & $\left(\phi^{\dagger} i \stackrel{\leftrightarrow}{a} D_{\mu} \phi\right)\left(\bar{l} \tau^{a} \gamma^{\mu} l\right)$ \\
\hline $\mathcal{O}_{\phi l}^{(1)}$ & $\left(\phi^{\dagger} i \stackrel{\leftrightarrow}{D}_{\mu} \phi\right)\left(\bar{l}^{a} \tau^{\mu} \gamma^{\mu} l\right)$ & & & & \\
\hline
\end{tabular}

At NLO, there are 22 additional operators that contribute:

\begin{tabular}{|c|c|c|c|c|c|c|c|c|}
\hline $\mathcal{O}_{e e}$ & $\mathcal{O}_{e u}$ & $\mathcal{O}_{l u}$ & $\mathcal{O}_{l d}$ & $\mathcal{O}_{l e}$ & $\mathcal{O}_{l q}^{(1)}$ & $\mathcal{O}_{l q}^{(3)}$ & $\mathcal{O}_{\phi B}$ & $\mathcal{O}_{\phi W}$ \\
\hline $\mathcal{O}_{u B}$, & $\mathcal{O}_{u W}$, & $\mathcal{O}_{W}$, & $\mathcal{O}_{q d}^{(1)}$ & $\mathcal{O}_{q q}^{(3)}$, & $\mathcal{O}_{q q}^{(1)}$, & $\mathcal{O}_{q u}^{(1)}$, & $\mathcal{O}_{u d}^{(1)}$ & $\mathcal{O}_{u u}$, \\
\hline
\end{tabular}

Definitions for these operators can be found in Refs. [30,31]. We use the Feynman rules in $R_{\xi}$ gauge from Ref. [32].

The SMEFT interactions cause the gauge field kinetic energies to have noncanonical normalizations and following Ref. [32], we define "barred" fields and couplings,

$$
\begin{aligned}
\bar{W}_{\mu}^{a} & \equiv\left(1-\mathcal{C}_{\phi W} v^{2} / \Lambda^{2}\right) W_{\mu}^{a} \\
\bar{B}_{\mu} & \equiv\left(1-\mathcal{C}_{\phi B} v^{2} / \Lambda^{2}\right) B_{\mu} \\
\bar{g}_{2} & \equiv\left(1+\mathcal{C}_{\phi W} v^{2} / \Lambda^{2}\right) g_{2} \\
\bar{g}_{1} & \equiv\left(1+\mathcal{C}_{\phi B} v^{2} / \Lambda^{2}\right) g_{1},
\end{aligned}
$$

such that $\bar{W}_{\mu} \bar{g}_{2}=W_{\mu} g_{2}$ and $\bar{B}_{\mu} \bar{g}_{1}=B_{\mu} g_{1}$. The barred fields have canonically normalized kinetic energy interactions. The masses of the $\mathrm{W}$ and $\mathrm{Z}$ fields to $\mathcal{O}\left(\frac{1}{\Lambda^{2}}\right)$ are $[32,33]$,

$$
\begin{aligned}
& M_{W}^{2}=\frac{\bar{g}_{2}^{2} v^{2}}{4}, \\
& M_{Z}^{2}=\frac{\left(\bar{g}_{1}^{2}+\bar{g}_{2}^{2}\right) v^{2}}{4}+\frac{v^{4}}{\Lambda^{2}}\left(\frac{1}{8}\left(\bar{g}_{1}^{2}+\bar{g}_{2}^{2}\right) \mathcal{C}_{\phi D}+\frac{1}{2} \bar{g}_{1} \bar{g}_{2} \mathcal{C}_{\phi W B}\right) .
\end{aligned}
$$

Dimension-6 4-fermion operators give contributions to the decay of the $\mu$, changing the relation between the vacuum expectation value (vev), $v$, and the Fermi constant $G_{\mu}$,

$$
G_{\mu} \equiv \frac{1}{\sqrt{2} v^{2}}-\frac{1}{\sqrt{2} \Lambda^{2}} \mathcal{C}_{l l}+\frac{\sqrt{2}}{\Lambda^{2}} \mathcal{C}_{\phi l}^{(3)}
$$

The tree level SMEFT couplings of fermions to the $Z$ and $W$ are given in terms of our input parameters $\left(\alpha, M_{Z}, G_{\mu}\right)$,

$$
\begin{aligned}
L \equiv & 2 M_{Z} \sqrt{\sqrt{2} G_{\mu}} Z_{\mu}\left[g_{L}^{Z q}+\delta g_{L}^{Z q}\right] \bar{q} \gamma_{\mu} q+2 M_{Z} \sqrt{\sqrt{2} G_{\mu}} Z_{\mu}\left[g_{R}^{Z u}+\delta g_{R}^{Z u}\right] \bar{u}_{R} \gamma_{\mu} u_{R} \\
& +2 M_{Z} \sqrt{\sqrt{2} G_{\mu}} Z_{\mu}\left[g_{R}^{Z d}+\delta g_{R}^{Z d}\right] \bar{d}_{R} \gamma_{\mu} d_{R}+2 M_{Z} \sqrt{\sqrt{2} G_{\mu}} Z_{\mu}\left[g_{L}^{Z l}+\delta g_{L}^{Z l}\right] \bar{l}_{\mu} l \\
& +2 M_{Z} \sqrt{\sqrt{2} G_{\mu}} Z_{\mu}\left[g_{R}^{Z e}+\delta g_{R}^{Z e}\right] \bar{e}_{R} \gamma_{\mu} e_{R}+2 M_{Z} \sqrt{\sqrt{2} G_{\mu}}\left(\delta g_{R}^{Z \nu}\right) \bar{\nu}_{R} \gamma_{\mu} \nu_{R} \\
& +\frac{\bar{g}_{2}}{\sqrt{2}}\left\{W_{\mu}\left[\left(1+\delta g_{L}^{W q}\right) \bar{u}_{L} \gamma_{\mu} d_{L}+\left(\delta g_{R}^{W q}\right) \bar{u}_{R} \gamma_{\mu} d_{R}\right]\right. \\
& \left.+W_{\mu}\left[\left(1+\delta g_{L}^{W l}\right) \bar{\nu}_{L} \gamma_{\mu} e_{L}+\left(\delta g_{R}^{W \nu}\right) \bar{\nu}_{R} \gamma_{\mu} e_{R}\right]+\text { H.c. }\right\} .
\end{aligned}
$$

We assume all couplings are flavor independent and we neglect Cabbibo-Kobayashi-Maskawa (CKM) mixing. The weak coupling in Eq. (6) is evaluated using the LO SM relation and Eq. (7) serves as the definition of $s_{W}^{2}$,

$$
\begin{aligned}
& \bar{g}_{2}^{2}=2 \sqrt{2} G_{\mu} M_{Z}^{2}\left(1+\sqrt{1-\frac{4 \pi \alpha}{\sqrt{2} G_{\mu} M_{Z}^{2}}}\right) . \\
& s_{W}^{2} \equiv \frac{4 \pi \alpha}{\bar{g}_{2}^{2}} .
\end{aligned}
$$

Since we are working to $\mathcal{O}\left(\frac{v^{2}}{\Lambda^{2}}\right)$, we omit dipole type operators that do not interfere with the SM contributions to $Z$ and $W$ pole observables. Similarly, the contributions from right-handed $W$ couplings and the right-handed $Z \bar{\nu} \nu$ interaction do not contribute to our study. The tree level couplings are,

$$
g_{R}^{Z f}=-s_{W}^{2} Q_{f} \quad \text { and } \quad g_{L}^{Z f}=T_{3}^{f}-s_{W}^{2} Q_{f}
$$


with $T_{3}^{f}= \pm \frac{1}{2} . S U(2)$ invariance implies,

$$
\begin{gathered}
\delta g_{L}^{W q}=\delta g_{L}^{Z u}-\delta g_{L}^{Z d} \\
\delta g_{L}^{W l}=\delta g_{L}^{Z \nu}-\delta g_{L}^{Z e} .
\end{gathered}
$$

$$
M_{W}, \quad \Gamma_{W}, \quad \Gamma_{Z}, \quad \sigma_{h}, \quad R_{l}, \quad A_{l, F B}, \quad R_{b},
$$

The SM results for these observables are quite precisely known, and as a by product of our study we recover the known NLO QCD and NLO EW results as a check of our calculation [35].

The next-to-leading order contributions to $Z$ and $W$ pole observables require the calculation of one loop virtual diagrams in the SMEFT and in most cases, the contribution also of real photon and gluon emission diagrams. Since the SMEFT theory is renormalizable order by order in the $\left(v^{2} / \Lambda^{2}\right)$ expansion, we retain only terms of $\mathcal{O}\left(v^{2} / \Lambda^{2}\right)$. The one-loop SMEFT calculations contain both tree level and one-loop contributions from the dimension-6 operators, along with the full electroweak and QCD one-loop SM amplitudes. Sample diagrams contributing to the $Z$ decay widths at NLO are shown in Fig. 1. For $W$ decays, there are also dipole-like $\gamma(g) W f \bar{f}$ contact interactions that we include. (The corresponding $\gamma(g) Z f \bar{f}$ operators first arise at dimension-8.) Since we concentrate on the $Z$ pole physics, we calculate the cross sections, $e^{+} e^{-} \rightarrow$ hadrons, using the narrow width approximation:

$$
\sigma_{\mathrm{had}}^{0}=\sum_{f=u, d, s, c, b} \frac{12 \pi}{M_{Z}^{2}} \frac{\Gamma_{e} \Gamma_{f}}{\Gamma_{Z}^{2}} .
$$

\begin{tabular}{|c|c|}
\hline & Warsaw Basis \\
\hline$\delta g_{L}^{Z u}$ & $-\frac{v^{2}}{2 \Lambda^{2}}\left(\mathcal{C}_{\phi q}^{(1)}-\mathcal{C}_{\phi q}^{(3)}\right)+\frac{1}{2} \delta g_{Z}+\frac{2}{3}\left(\delta s_{W}^{2}-s_{W}^{2} \delta g_{Z}\right)$ \\
\hline$\delta g_{L}^{Z d}$ & $-\frac{v^{2}}{2 \Lambda^{2}}\left(\mathcal{C}_{\phi q}^{(1)}+\mathcal{C}_{\phi q}^{(3)}\right)-\frac{1}{2} \delta g_{Z}-\frac{1}{3}\left(\delta s_{W}^{2}-s_{W}^{2} \delta g_{Z}\right)$ \\
\hline$\delta g_{L}^{Z \nu}$ & $-\frac{v^{2}}{2 \Lambda^{2}}\left(\mathcal{C}_{\phi l}^{(1)}-\mathcal{C}_{\phi l}^{(3)}\right)+\frac{1}{2} \delta g_{Z}$ \\
\hline$\delta g_{L}^{Z e}$ & $-\frac{v^{2}}{2 \Lambda^{2}}\left(\mathcal{C}_{\phi l}^{(1)}+\mathcal{C}_{\phi l}^{(3)}\right)-\frac{1}{2} \delta g_{Z}-\left(\delta s_{W}^{2}-s_{W}^{2} \delta g_{Z}\right)$ \\
\hline$\delta g_{R}^{Z u}$ & $-\frac{v^{2}}{2 \Lambda^{2}} \mathcal{C}_{\phi u}+\frac{2}{3}\left(\delta s_{W}^{2}-s_{W}^{2} \delta g_{Z}\right)$ \\
\hline$\delta g_{R}^{Z d}$ & $-\frac{v^{2}}{2 \Lambda^{2}} \mathcal{C}_{\phi d}-\frac{1}{3}\left(\delta s_{W}^{2}-s_{W}^{2} \delta g_{Z}\right)$ \\
\hline$\delta g_{R}^{Z e}$ & $-\frac{v^{2}}{2 \Lambda^{2}} \mathcal{C}_{\phi e}-\left(\delta s_{W}^{2}-s_{W}^{2} \delta g_{Z}\right)$ \\
\hline$\delta g_{L}^{W q}$ & $\frac{v^{2}}{\Lambda^{2}} \mathcal{C}_{\phi q}^{(3)}+c_{W}^{2} \delta g_{Z}+\delta s_{W}^{2}$ \\
\hline$\delta g_{L}^{W l}$ & $\frac{v^{2}}{\Lambda^{2}} \mathcal{C}_{\phi l}^{(3)}+c_{W}^{2} \delta g_{Z}+\delta s_{W}^{2}$ \\
\hline$\delta g_{Z}$ & $-\frac{v^{2}}{\Lambda^{2}}\left(\delta v+\frac{1}{4} \mathcal{C}_{\phi D}\right)$ \\
\hline$\delta v$ & $\mathcal{C}_{\phi l}^{(3)}-\frac{1}{2} \mathcal{C}_{l l}$ \\
\hline$\delta s_{W}^{2}$ & 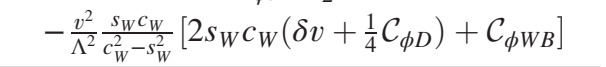 \\
\hline
\end{tabular}

TABLE II. Anomalous fermion couplings at LO in the Warsaw [31] basis.
The SMEFT contributions to the effective couplings are listed in Table II [34].

\section{III. $W$ AND $Z$ POLE OBSERVABLES TO NLO}

The observables we consider are

$R_{c}, \quad A_{F B, b}, \quad A_{F B, c}, \quad A_{b}, \quad A_{c}, \quad A_{l}$.

Corrections to this formula are of higher order and we do not include them [36]. Nonresonant contributions, such as photon exchange, box diagrams and 4-fermions interactions [34], are also not included because they do not contribute to the observables on the $Z$ pole to $\mathcal{O}\left(\frac{1}{\Lambda^{2}}\right)$.

We employ a modified on-shell (OS) scheme, where the SM parameters are renormalized in the OS scheme. The effective field theory coefficients of the dimension- 6 operators are treated as $\overline{\mathrm{MS}}$ parameters and the poles of the oneloop coefficients $\mathcal{C}_{i}$ are known from Refs. [33,37,38],

$$
\mathcal{C}_{i}(\mu)=\mathcal{C}_{0, i}-\frac{1}{2 \hat{\epsilon}} \frac{1}{16 \pi^{2}} \gamma_{i j} \mathcal{C}_{j}
$$

where $\mu$ is the renormalization scale, $\gamma_{i j}$ is the one-loop anomalous dimension,

$$
\mu \frac{d \mathcal{C}_{i}}{d \mu}=\frac{1}{16 \pi^{2}} \gamma_{i j} \mathcal{C}_{j}
$$

and $\hat{\epsilon}^{-1} \equiv \epsilon^{-1}-\gamma_{E}+\log (4 \pi)$.

The renormalized SM gauge boson masses are,

$$
M_{V}^{2}=M_{0, V}^{2}-\Pi_{V V}\left(M_{V}^{2}\right),
$$

where $\Pi_{V V}\left(M_{V}^{2}\right)$ is the one-loop correction to the 2-point function for $\mathrm{Z}$ or $\mathrm{W}$ computed on-shell and tree level quantities are denoted with the subscript 0 in this section. The gauge boson 2- point functions in the SMEFT can be found analytically in Refs. [39,40]. The one-loop relation between the vacuum expectation value and the Fermi constant is,

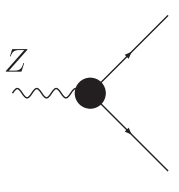

(a)

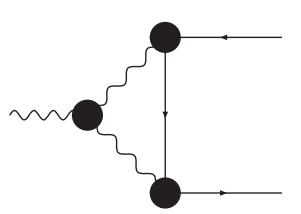

(b)

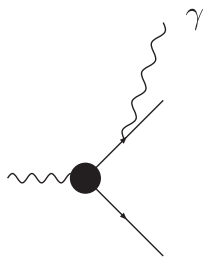

(c)

FIG. 1. Sample electroweak diagrams contributing to $Z \rightarrow f \bar{f}$ at NLO in the SMEFT: (a) Tree level SMEFT diagram, (b) virtual SMEFT diagram, and (c) real photon emission in the SMEFT. The circles represent potential insertions of dimension-6 SMEFT operators. 


$$
G_{\mu}+\frac{\mathcal{C}_{l l}}{\sqrt{2} \Lambda^{2}}-\sqrt{2} \frac{\mathcal{C}_{\phi l}^{(3)}}{\Lambda^{2}} \equiv \frac{1}{\sqrt{2} v_{0}^{2}}(1+\Delta r),
$$

where $v_{0}$ is the unrenormalized minimum of the potential and $\Delta r$ is obtained from the one-loop corrections to $\mu$ decay. Complete analytic expressions for $\Delta r$ in both the SM and the SMEFT at dimension-6 are given in Ref. [24]. Finally, the on-shell renormalization of $\alpha$ is extracted from the renormalization of the $\bar{l} l \gamma$ vertex.

We obtain the relevant amplitudes for the virtual contributions using FEYNARTS [41] with a model file generated by FEYNRULES [42] and the Feynman rules of Ref. [32]. Then we use FEYNCALC $[43,44]$ to manipulate and reduce the integrals and LOOPTOOLS [45] for the numerical evaluation.

The $Z$ decays to charged fermions receive contributions from one-loop virtual diagrams and from real photon emission that are separately IR divergent and we regulate these divergences with a photon mass. Since we only consider the inclusive quantities of Eq. (10), the photon mass dependence cancels after integration over the photon phase space and there is no need for a photon energy cut. The complicated form of the SMEFT vertices makes direct integration of the phase space difficult, so we use the method of Ref. [46], where the integration over the photon phase space is replaced with a loop integration. This is possible after we use the identity,

$$
2 i \pi \delta\left(p^{2}-m^{2}\right)=\frac{1}{p^{2}-m^{2}+i 0}-\frac{1}{p^{2}-m^{2}-i 0} .
$$

After making this replacement, we treat the momenta of the outgoing particles as internal loop momenta, the integration over the phase space becomes an integration over the loop momenta and we can use the IBP relations to reduce the loop integrals to known master integrals. In the case of $Z \rightarrow \bar{f} \gamma \gamma$, the integrals are 2-point 2-loop integrals, for which a generic basis of master integrals is known $[47,48]$ and the reduction can be done using FIRE [49]. This is identical to the technique we applied in the calculation of the real contributions to $H \rightarrow W^{+} W^{-} \gamma$ in Ref. [20].

\section{RESULTS}

We take as our physical input parameters,

$$
\begin{aligned}
G_{\mu} & =1.1663787(6) \times 10^{-5} \mathrm{GeV}^{-2} \\
M_{Z} & =91.1876 \pm .0021 \mathrm{GeV} \\
\frac{1}{\alpha} & =137.035999139(31) \\
\Delta \alpha_{\text {had }}^{(5)} & =0.02764 \pm 0.00009 \\
\alpha_{s}\left(M_{Z}\right) & =0.1181 \pm 0.0011 \\
M_{H} & =125.10 \pm 0.14 \mathrm{GeV} \\
M_{t} & =172.9 \pm 0.5 \mathrm{GeV} .
\end{aligned}
$$

The lowest order SMEFT contributions to $\mathrm{Z}$ pole observables, $\mathcal{O}_{i}$ are well known. We write the SMEFT predictions for the observables as,

$$
\begin{aligned}
O_{i}^{\mathrm{SMEFT}, \mathrm{LO}} & =O_{i}^{\mathrm{SM}}+\delta O_{i}^{\mathrm{LO}} \\
O_{i}^{\mathrm{SMEFT}, \mathrm{NLO}} & =O_{i}^{\mathrm{SM}}+\delta O_{i}^{\mathrm{NLO}},
\end{aligned}
$$

and we present our results numerically. In Table III, we summarize the current state of the SM theory and the experimental results. The theory errors include the parametric uncertainties on $M_{t}$ and $M_{H}$ [36]. In evaluating $O_{i}^{\mathrm{SM}}$ in Eq. (17), we always use the most accurately calculated value given in Table III.

We do not include the effective weak leptonic mixing angle in our fit since it can be directly derived from other observables, but present it here for completeness.

$$
\begin{aligned}
\delta \sin ^{2} \theta_{l, \mathrm{eff}}^{\mathrm{LO}}= & \frac{v^{2}}{\Lambda^{2}}\left\{-0.28785 \mathcal{C}_{\phi e}-0.21215 \mathcal{C}_{\phi l}^{(1)}+0.36851 \mathcal{C}_{\phi l}^{(3)}-0.29033 \mathcal{C}_{l l}+0.14517 \mathcal{C}_{\phi D}+0.71015 \mathcal{C}_{\phi W B}\right\} \\
\delta \sin ^{2} \theta_{l, \mathrm{eff}}^{\mathrm{NLO}}= & \frac{v^{2}}{\Lambda^{2}}\left\{-0.2726 \mathcal{C}_{\phi e}-0.23666 \mathcal{C}_{\phi l}^{(1)}+0.42246 \mathcal{C}_{\phi l}^{(3)}-0.31904 \mathcal{C}_{l l}+0.16629 \mathcal{C}_{\phi D}+0.77518 \mathcal{C}_{\phi W B}\right. \\
& -0.00036 \mathcal{C}_{e d}-0.00100 \mathcal{C}_{e e}+0.00677 \mathcal{C}_{e u}+0.00161 \mathcal{C}_{\phi d}+0.01033 \mathcal{C}_{\phi q}^{(1)} \\
& -0.00871 \mathcal{C}_{\phi q}^{(3)}-0.01424 \mathcal{C}_{\phi u}-0.00028 \mathcal{C}_{l d}-0.00064 \mathcal{C}_{l e}-0.00401 \mathcal{C}_{l q}^{(1)} \\
& -0.00106 \mathcal{C}_{l q}^{(3)}+0.00531 \mathcal{C}_{l u}+0.00032 \mathcal{C}_{\phi B}+0.00004 \mathcal{C}_{\phi \square}+0.00032 \mathcal{C}_{\phi W} \\
& \left.-0.00512 \mathcal{C}_{q e}+0.01087 \mathcal{C}_{u B}+0.00917 \mathcal{C}_{u W}+0.00053 \mathcal{C}_{W}\right\}
\end{aligned}
$$

The NLO corrections to $\sin ^{2} \theta_{l \text {,eff }}$ change the numerical effects of the coefficients appearing at tree level by $\mathcal{O}(5-10 \%)$, and introduce dependencies on other coefficients. 
For the $W$ mass and total width, we find the predictions,

$$
\begin{aligned}
\delta M_{W}^{\mathrm{LO}}= & \frac{v^{2}}{\Lambda^{2}}\left\{-29.827 \mathcal{C}_{\phi l}^{(3)}+14.914 \mathcal{C}_{l l}-27.691 \mathcal{C}_{\phi D}-57.479 \mathcal{C}_{\phi W B}\right\} \\
\delta M_{W}^{\mathrm{NLO}}= & \frac{v^{2}}{\Lambda^{2}}\left\{-35.666 \mathcal{C}_{\phi l}^{(3)}+17.243 \mathcal{C}_{l l}-30.272 \mathcal{C}_{\phi D}-64.019 \mathcal{C}_{\phi W B}-0.137 \mathcal{C}_{\phi d}-0.137 \mathcal{C}_{\phi e}-0.166 \mathcal{C}_{\phi l}^{(1)}-2.032 \mathcal{C}_{\phi q}^{(1)}\right. \\
& \left.+1.409 \mathcal{C}_{\phi q}^{(3)}+2.684 \mathcal{C}_{\phi u}+0.438 \mathcal{C}_{l q}^{(3)}-0.027 \mathcal{C}_{\phi B}-0.033 \mathcal{C}_{\phi \square}-0.035 \mathcal{C}_{\phi W}-0.902 \mathcal{C}_{u B}-0.239 \mathcal{C}_{u W}-0.15 \mathcal{C}_{W}\right\} \\
\delta \Gamma_{W}^{\mathrm{LO}}= & \frac{v^{2}}{\Lambda^{2}}\left\{-5.092 \mathcal{C}_{\phi l}^{(3)}+2.784 \mathcal{C}_{\phi q}^{(3)}+3.242 \mathcal{C}_{l l}-2.143 \mathcal{C}_{\phi D}-4.448 \mathcal{C}_{\phi W B}\right\} \\
\delta \Gamma_{W}^{\mathrm{NLO}}= & \frac{v^{2}}{\Lambda^{2}}\left\{-5.597 \mathcal{C}_{\phi l}^{(3)}+3.019 \mathcal{C}_{\phi q}^{(3)}+3.361 \mathcal{C}_{l l}-2.276 \mathcal{C}_{\phi D}-4.811 \mathcal{C}_{\phi W B}-0.01 \mathcal{C}_{\phi d}-0.01 \mathcal{C}_{\phi e}-0.017 \mathcal{C}_{\phi l}^{(1)}-0.153 \mathcal{C}_{\phi q}^{(1)}\right. \\
& +0.203 \mathcal{C}_{\phi u}+0.048 \mathcal{C}_{l q}^{(3)}-0.002 \mathcal{C}_{\phi B}-0.003 \mathcal{C}_{\phi \square}-0.004 \mathcal{C}_{\phi W}-0.03 \mathcal{C}_{q q}^{(1)}-0.094 \mathcal{C}_{q q}^{(3)}-0.068 \mathcal{C}_{u B} \\
& \left.-0.014 \mathcal{C}_{u W}-0.013 \mathcal{C}_{W}\right\}
\end{aligned}
$$

It is interesting to note that some of the contributions to the $W$ mass and width change by more than $10 \%$ when going from LO to NLO in the SMEFT. The NLO SMEFT contributions to the other observables of Eq. (10) given in Sec. IV.

We fit to the experimental data given in Table III, (omitting $\sin ^{2} \theta_{\text {eff }}$ ) since it can be directly derived from other observables). The most accurate SM predictions are given in the right-hand column and we use these values in our fits, as opposed to the LO or NLO SM contributions directly calculated. The pole observables we consider are [60-62]:

$$
M_{W}, \quad \Gamma_{W}, \quad \Gamma_{Z}, \quad \sigma_{h}, \quad R_{l}, \quad A_{l, F B}, \quad R_{b}, \quad R_{c}, \quad A_{F B, b}, \quad A_{F B, c}, \quad A_{b}, \quad A_{c}, \quad A_{l},
$$

where we assume lepton universality and the experimental correlations can be found in Ref. [53]. We include the measurements of $A_{l}$ from LEP and SLD as separate data points.

The $\chi^{2}$ is computed from,

$$
\chi^{2}=\Sigma_{i, j}\left(O_{i}^{\exp }-O_{i}^{\mathrm{SMEFT}}\right) \sigma_{i j}^{-2}\left(O_{j}^{\mathrm{exp}}-O_{j}^{\mathrm{SMEFT}}\right) .
$$

TABLE III. Experimental results and SM predictions for $W$ and $Z$ pole observables, assuming lepton universality The theory includes the full set of 2-loop contributions for the $Z$ pole observables, along with higher order corrections when known. When not specified, the numbers are taken from Table 10.5 of the electroweak review of Ref. [50]. The theory predictions are computed using the formulas in the indicated references and our input parameters, and the theory errors include the parametric uncertainties on $M_{t}$ and $M_{H}$ [36], along with the estimated theory uncertainties described in the respective papers.

\begin{tabular}{lcc}
\hline \hline Measurement & Experiment & "Best" theory \\
\hline$\Gamma_{Z}(\mathrm{GeV})$ & $2.4952 \pm 0.0023$ & $2.4945 \pm 0.0006[36,51,52]$ \\
$\sigma_{h}(\mathrm{nb})$ & $41.540 \pm 0.037$ & $41.491 \pm 0.008[36,51,52]$ \\
$R_{l}$ & $20.767 \pm 0.025[53]$ & $20.749 \pm 0.009[36,51,52]$ \\
$R_{b}$ & $0.21629 \pm 0.00066$ & $0.21586 \pm 0.0001[36,51,52]$ \\
$R_{c}$ & $0.1721 \pm 0.0030$ & $0.17221 \pm 0.00005[36,51,52]$ \\
$A_{l}$ & $0.1465 \pm 0.0033[53]$ & $0.1472 \pm 0.0004[36,54]$ \\
$A_{c}$ & $0.670 \pm 0.027$ & $0.6679 \pm 0.0002[36,54]$ \\
$A_{b}$ & $0.923 \pm 0.020$ & $0.92699 \pm 0.00006[36,54,55]$ \\
$A_{l, F B}$ & $0.0171 \pm 0.0010$ & $0.0162 \pm 0.0001[36,54]$ \\
$A_{b, F B}$ & $0.0992 \pm 0.0016$ & $0.1023 \pm 0.0003[36,54,55]$ \\
$A_{c, F B}$ & $0.0707 \pm 0.0035$ & $0.0737 \pm 0.0003[36,54]$ \\
$A_{l}(S L D)$ & $0.1513 \pm 0.0021[53]$ & $0.1472 \pm 0.0004[36,54]$ \\
$\sin ^{2} \theta_{l, \mathrm{eff}}$ & $0.23179 \pm 0.00035[56]$ & $0.23150 \pm 0.00006[36,54,55]$ \\
$M_{W}(\mathrm{GeV})$ & $80.379 \pm 0.012[50]$ & $80.359 \pm 0.006[57,58]$ \\
$\Gamma_{W}(\mathrm{GeV})$ & $2.085 \pm 0.042[50]$ & $2.0904 \pm 0.0003[59]$ \\
\hline \hline
\end{tabular}


Using the LO SMEFT expressions for the observables and taking $\Lambda=1 \mathrm{TeV}$, we find, ${ }^{1}$

$$
\begin{aligned}
\chi_{\mathrm{LO}}^{2}= & \chi_{\mathrm{SM}}^{2}+32 \mathcal{C}_{\phi d}+105 \mathcal{C}_{\phi e}-445 \mathcal{C}_{\phi l}^{(1)}+639 \mathcal{C}_{\phi l}^{(3)}-49 \mathcal{C}_{\phi q}^{(1)}-60 \mathcal{C}_{\phi q}^{(3)}-11 \mathcal{C}_{\phi u}-424 \mathcal{C}_{l l}+491 \mathcal{C}_{\phi D} \\
& +1114 \mathcal{C}_{\phi W B}+\vec{C}_{\mathrm{LO}}^{T} M_{\mathrm{LO}} \vec{C}_{\mathrm{LO}}
\end{aligned}
$$

where

$$
\vec{C}_{\mathrm{LO}}^{T}=\left(\mathcal{C}_{l l}, \mathcal{C}_{\phi W B}, \mathcal{C}_{\phi u}, \mathcal{C}_{\phi q}^{(3)}, \mathcal{C}_{\phi q}^{(1)}, \mathcal{C}_{\phi l}^{(3)}, \mathcal{C}_{\phi l}^{(1)}, \mathcal{C}_{\phi e}, \mathcal{C}_{\phi D}, \mathcal{C}_{\phi d}\right)
$$

and we find $\chi_{\mathrm{SM}}^{2} \sim 13.42$. The symmetric matrix $M_{\mathrm{LO}}$ is,

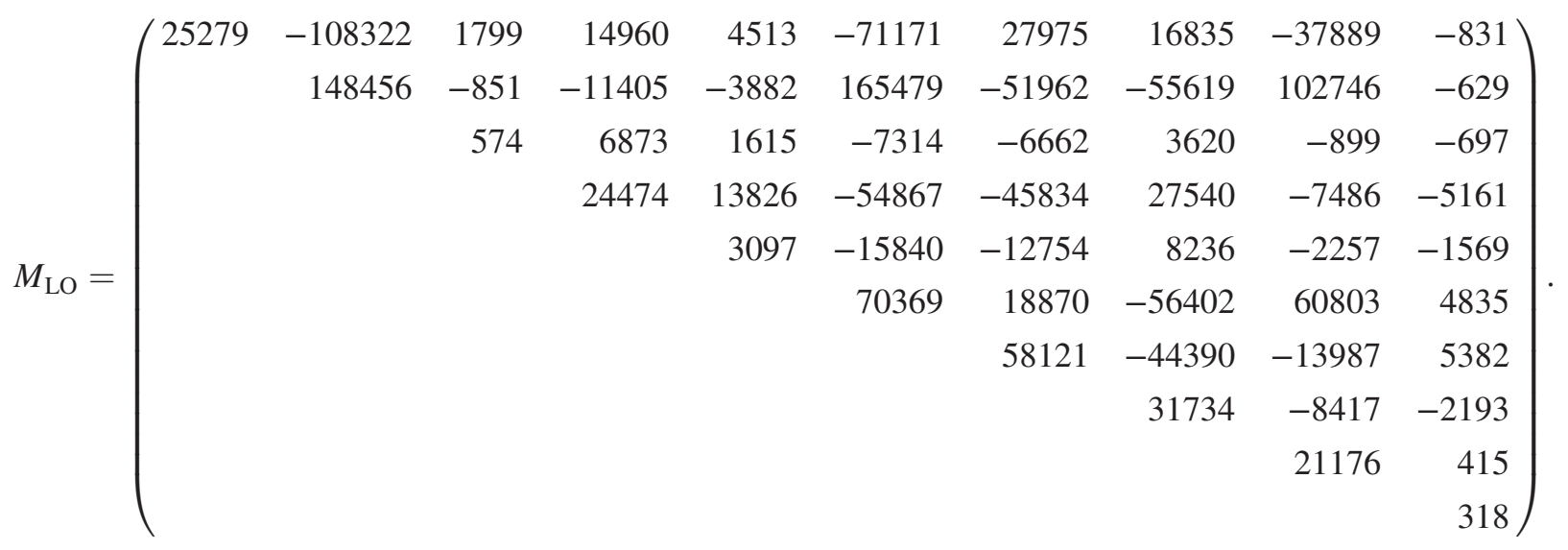

Using the NLO SMEFT expressions we find $\chi_{\mathrm{NLO}}^{2}$, (for $\Lambda=1 \mathrm{TeV}$ ),

$$
\begin{aligned}
\chi_{\mathrm{NLO}}^{2}= & \chi_{\mathrm{SM}}^{2}-403 \mathcal{C}_{l l}+1070 \mathcal{C}_{\phi W B}-53 \mathcal{C}_{\phi u}-93 \mathcal{C}_{\phi q}^{(3)}-19 \mathcal{C}_{\phi q}^{(1)}+667 \mathcal{C}_{\phi l}^{(3)}-402 \mathcal{C}_{\phi l}^{(1)}+176 \mathcal{C}_{\phi e}+503 \mathcal{C}_{\phi D}+27 \mathcal{C}_{\phi d} \\
& -1.48 \mathcal{C}_{q q}^{(1)}+0.55 \mathcal{C}_{\phi \square}+0.62 \mathcal{C}_{\phi W}+0.48 \mathcal{C}_{\phi B}+6.55 \mathcal{C}_{u W}+15 \mathcal{C}_{u B}+0.23 \mathcal{C}_{e d}+0.063 \mathcal{C}_{d d}+0.56 \mathcal{C}_{e e}+1.40 \mathcal{C}_{q q}^{(3)} \\
& +2.38 \mathcal{C}_{W}+0.53 \mathcal{C}_{u u}-0.54 \mathcal{C}_{u d}^{(1)}+1.05 \mathcal{C}_{q u}^{(1)}-4.88 \mathcal{C}_{l q}^{(3)}+2.8 \mathcal{C}_{q e}+0.34 \mathcal{C}_{q d}^{(1)}+9.8 \mathcal{C}_{l u}-0.32 \mathcal{C}_{l e}-0.49 \mathcal{C}_{l d} \\
& -3.8 \mathcal{C}_{e u}-7.4 \mathcal{C}_{l q}^{(1)}+\vec{C}_{\mathrm{NLO}}^{T} M_{\mathrm{NLO}} \vec{C}_{\mathrm{NLO}}
\end{aligned}
$$

where,

$$
\begin{aligned}
\vec{C}_{\mathrm{NLO}}^{T}= & \left(\mathcal{C}_{l l}, \mathcal{C}_{\phi W B}, \mathcal{C}_{\phi u}, \mathcal{C}_{\phi q}^{(3)}, \mathcal{C}_{\phi q}^{(1)}, \mathcal{C}_{\phi l}^{(3)}, \mathcal{C}_{\phi l}^{(1)}, \mathcal{C}_{\phi e}, \mathcal{C}_{\phi D}, \mathcal{C}_{\phi d},\right. \\
& \mathcal{C}_{e d}, \mathcal{C}_{e e}, \mathcal{C}_{e u}, \mathcal{C}_{l u}, \mathcal{C}_{l d}, \mathcal{C}_{l e}, \mathcal{C}_{l q}^{(1)}, \mathcal{C}_{l q}^{(3)}, \mathcal{C}_{\phi B}, \mathcal{C}_{\phi W}, \mathcal{C}_{\phi \square}, \\
& \left.\mathcal{C}_{q e}, \mathcal{C}_{u B}, \mathcal{C}_{u W}, \mathcal{C}_{W}, \mathcal{C}_{q d}^{(1)}, \mathcal{C}_{q q}^{(3)}, \mathcal{C}_{q q}^{(1)}, \mathcal{C}_{q u}^{(1)}, \mathcal{C}_{u d}^{(1)}, \mathcal{C}_{u u}, \mathcal{C}_{d d}\right),
\end{aligned}
$$

where the numerical form of $M_{\mathrm{NLO}}$ is given in the Supplemental Material [65]. At NLO, the $\chi^{2}$ now depends on 32 coefficients, and the effects of the coefficients appearing at LO have shifted by 5\%-10\%. The (relatively) large shift of the coefficients of $\mathcal{C}_{\phi u}$ and $\mathcal{C}_{\phi Q}^{(1,3)}$ are due to the top quark loop.

To study the numerical importance of the NLO effects, we begin by keeping only one coefficient nonzero at a time. We find the 95\% confidence level regions at LO and NLO shown in Tables IV and V. The largest effect of the NLO corrections is on the coefficient of $\mathcal{C}_{\phi u}$. The relatively large allowed values for $\mathcal{C}_{\phi d}$ are the result of the discrepancy in the measured value $A_{F B, b}$ from the SM prediction.

\footnotetext{
${ }^{1}$ Our results are consistent with SMEFT fits to purely LEP observables using slightly different sets of inputs [9,60-62].
} 
TABLE IV. 95\% confidence level allowed ranges for single parameter fit to coefficients contributing to the lowest order predictions. The scale $\Lambda$ is taken to be $1 \mathrm{TeV}$.

\begin{tabular}{lcc}
\hline \hline Coefficient & LO & NLO \\
\hline $\mathcal{C}_{l l}$ & {$[-0.0039,0.021]$} & {$[-0.0044,0.019]$} \\
$\mathcal{C}_{\phi W B}$ & {$[-0.0088,0.0013]$} & {$[-0.0079,0.0016]$} \\
$\mathcal{C}_{\phi u}$ & {$[-0.072,0.091]$} & {$[-0.035,0.084]$} \\
$\mathcal{C}_{\phi q}^{(3)}$ & {$[-0.011,0.014]$} & {$[-0.010,0.014]$} \\
$\mathcal{C}_{\phi q}^{(1)}$ & {$[-0.027,0.043]$} & {$[-0.031,0.036]$} \\
$\mathcal{C}_{\phi l}^{(3)}$ & {$[-0.012,0.0029]$} & {$[-0.010,0.0028]$} \\
$\mathcal{C}_{\phi l}^{(1)}$ & {$[-0.0043,0.012]$} & {$[-0.0047,0.012]$} \\
$\mathcal{C}_{\phi e}$ & {$[-0.013,0.0094]$} & {$[-0.013,0.0080]$} \\
$\mathcal{C}_{\phi D}$ & {$[-0.025,0.0019]$} & {$[-0.023,0.0023]$} \\
$\mathcal{C}_{\phi d}$ & {$[-0.16,0.060]$} & {$[-0.13,0.063]$} \\
\hline \hline
\end{tabular}

At lowest order, the $\chi_{\mathrm{LO}}^{2}$ is sensitive to 8 combinations of operators, implying that there are 2 blind directions [6264]. These 8 combinations can be thought of as the combinations of operators contributing to $\delta g_{L}^{Z u}, \delta g_{L}^{Z d}$, $\delta g_{L}^{Z e}, \delta g_{L}^{Z \nu}, \delta g_{R}^{Z u}, \delta g_{R}^{Z d}, \delta g_{R}^{Z e}$, and $M_{W}$. Because of the $S U(2)$ symmetry of Eq. (9), at LO there is no additional information from $\Gamma_{W}$. Since our study includes only 14 data points, we clearly cannot fit to all of the SMEFT coefficients appearing at one loop At NLO, the fit is sensitive to only 10 combinations of operators. The additional information can be thought of as coming from $\delta g_{L}^{Z b}$ and $\Gamma_{W}$ where the top quark makes significant contributions. Since there are 32 coefficients that contribute to the NLO fit to the electroweak observables, resolving these 22 blind directions requires input from other processes and/or assumptions about which operators can be safely neglected.

We chose to perform our fits setting $\mathcal{C}_{\phi e}=0$ and $\mathcal{C}_{\phi q}^{(3)}=0$, along with setting all of the operators that first appear at NLO to 0 . We then marginalize over the remaining operators to study the numerical impacts of the NLO contributions. These results are shown in Table VI. We see that the effects of the NLO corrections
TABLE VI. 95\% confidence level allowed ranges for fit to coefficients marginalizing over the other 7 operators we are considering. The coefficients of all operators not listed in the table are set to 0 . The scale $\Lambda$ is taken to be $1 \mathrm{TeV}$.

\begin{tabular}{lcc}
\hline \hline Coefficient & LO & NLO \\
\hline $\mathcal{C}_{\phi D}$ & {$[-0.034,0.041]$} & {$[-0.039,0.051]$} \\
$\mathcal{C}_{\phi W B}$ & {$[-0.080,0.0021]$} & {$[-0.098,0.012]$} \\
$\mathcal{C}_{\phi d}$ & {$[-0.81,-0.093]$} & {$[-1.07,-0.03]$} \\
$\mathcal{C}_{\phi l}^{(3)}$ & {$[-0.025,0.12]$} & {$[-0.039,0.16]$} \\
$\mathcal{C}_{\phi u}$ & {$[-0.12,0.37]$} & {$[-0.21,0.41]$} \\
$\mathcal{C}_{\phi l}^{(1)}$ & {$[-0.0086,0.036]$} & {$[-0.0072,0.037]$} \\
$\mathcal{C}_{l l}$ & {$[-0.085,0.035]$} & {$[-0.087,0.033]$} \\
$\mathcal{C}_{\phi q}^{(1)}$ & {$[-0.060,0.076]$} & {$[-0.095,0.075]$} \\
\hline \hline
\end{tabular}

can be significant, although the numerical results are sensitive to which operators are set to 0 . Our results suggest that including the NLO corrections in the global fits (where the complete set of operators can potentially be bounded) may be important.

As another way of examining the impact of the NLO contributions, we consider the oblique parameters. The tree level SMEFT contributions are,

$$
\begin{aligned}
& \alpha \Delta S=4 c_{W} s_{W} \frac{v^{2}}{\Lambda^{2}} C_{\phi W B} \\
& \alpha \Delta T=-\frac{v^{2}}{2 \Lambda^{2}} \mathcal{C}_{\phi D} .
\end{aligned}
$$

For the NLO oblique parameter fit, we set all coefficients to 0 , except $\mathcal{C}_{\phi W B}$ and $\mathcal{C}_{\phi D}$. The resulting limits are shown in Fig. 2, (where what we are really plotting are the limits on the coefficients from a 2 parameter fit to our observables). In this example, the effect of the NLO SMEFT corrections is small. At NLO, new coefficients can influence the oblique parameters, and the complete one-loop SMEFT result is given in Ref. [39].

Our calculation includes only the resonant $Z$ and $W$ contributions to the precision electroweak observables. In

TABLE V. $\quad 95 \%$ confidence level allowed ranges for single parameter fit to coefficients not contributing to the lowest order predictions. The scale $\Lambda$ is taken to be $1 \mathrm{TeV}$.

\begin{tabular}{lccccc}
\hline \hline Coefficient & NLO & Coefficient & NLO & Coefficient & NLO \\
\hline $\mathcal{C}_{W}$ & {$[-4.8,0.48]$} & $\mathcal{C}_{u u}$ & {$[-1.1,0.99]$} & $\mathcal{C}_{u W}$ & {$[-0.78,0.29]$} \\
$\mathcal{C}_{u B}$ & {$[-0.57,0.11]$} & $\mathcal{C}_{q u}^{(1)}$ & {$[-2.2,1.3]$} & $\mathcal{C}_{q q}^{(3)}$ & {$[-0.32,0.29]$} \\
$\mathcal{C}_{q q}^{(1)}$ & {$[-0.93,1.49]$} & $\mathcal{C}_{q e}$ & {$[-0.75,0.48]$} & $\mathcal{C}_{q d}^{(1)}$ & {$[-9.8,5.0]$} \\
$\mathcal{C}_{\phi \square}$ & {$[-22,1.9]$} & $\mathcal{C}_{\phi W}$ & {$[-17,2.2]$} & $\mathcal{C}_{\phi B}$ & {$[-19,3.3]$} \\
$\mathcal{C}_{l u}$ & {$[-0.49,0.19]$} & $\mathcal{C}_{l q}^{(3)}$ & {$[-0.32,0.57]$} & $\mathcal{C}_{l q}^{(1)}$ & {$[-0.25,0.66]$} \\
$\mathcal{C}_{l e}$ & {$[-5.3,11]$} & $\mathcal{C}_{l d}$ & {$[-3.8,8.7]$} & $\mathcal{C}_{d d}$ & {$[-51,26]$} \\
$\mathcal{C}_{e d}$ & {$[-12,6.7]$} & $\mathcal{C}_{e e}$ & {$[-3.9,2.4]$} & $\mathcal{C}_{e u}$ & {$[-0.36,0.58]$} \\
$\mathcal{C}_{u d}^{(1)}$ & {$[-3.0,5.6]$} & & & & \\
\hline \hline
\end{tabular}




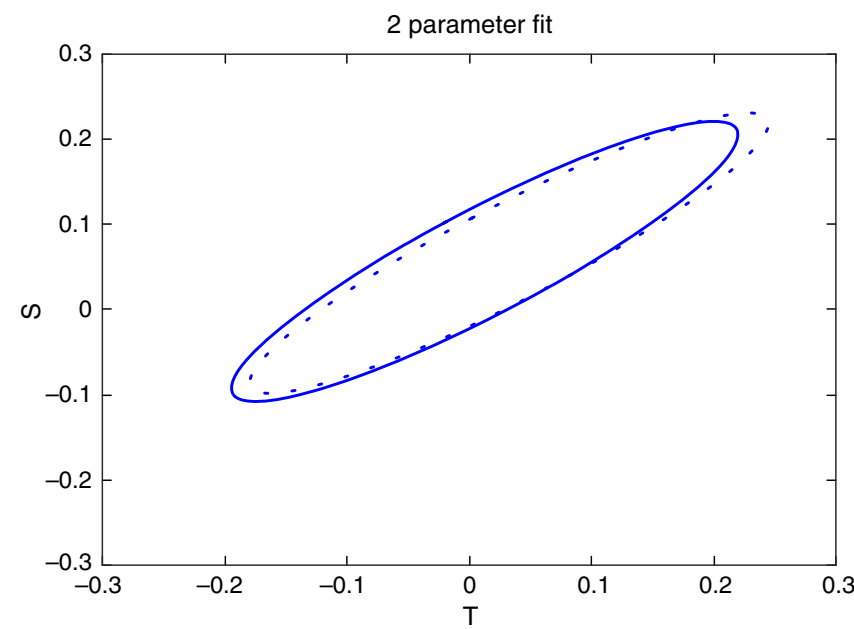

FIG. 2. $95 \%$ CL limits from a 2 parameter fit to $\mathcal{C}_{\phi W B}$ and $\mathcal{C}_{\phi D}$, setting all other coefficients to 0 . The scale $\Lambda=1 \mathrm{TeV}$.The solid line is the result of the LO fit, while the dotted line is the NLO fit to the electroweak parameters of this study.

the SMEFT, there are tree level nonresonant contributions due to 4-fermion operators that can complicate the experimental extraction of the widths from the data. The size of these effects has been estimated in Ref. [34]. For example,

$$
\delta \Gamma(Z \rightarrow \text { hadrons }) \sim 0.6 \mathrm{MeVC}_{4 f}\left(\frac{1 \mathrm{TeV}}{\Lambda}\right)^{2},
$$

where $C_{4 f}$ is a generic 4-fermion operator. These effects could potentially be similar in size to the electroweak corrections we have computed. There are also off-shell corrections proportional to $\frac{q^{2}}{\Lambda^{2}}$, where $q^{2}$ is the momentum running through the $Z$ boson propagator. The experimental cuts [53] were designed to extract predominantly the on- shell $Z$ events, so although the size of these effects is expected to be small a detailed theoretical study would be needed to quantify them.

\section{CONCLUSIONS}

We have computed the NLO electroweak and QCD corrections to the SMEFT predictions for the precision electroweak observables. Our results are presented in a numerical form that can easily be incorporated in the global fitting programs. We also present numerical results for the LO and NLO $\chi^{2}$ that can be customized for the reader's use. Our studies suggest that the NLO SMEFT corrections may have a sizable effect on the global fits. Numerical results for the SMEFT NLO expressions for the observables considered here, along with the $\chi_{\mathrm{LO}}^{2}, \chi_{\mathrm{NLO}}^{2}$ and the matrix $M_{\mathrm{NLO}}$, are posted at https://quark.phy.bnl.gov/Digital_Data_Archive/ dawson/ewpo_19.

\section{ACKNOWLEDGMENTS}

We thank Sam Homiller for discussions. S. D. is supported by the U.S. Department of Energy under Grant Contract No. de-sc0012704. P. P. G. is supported by the Spanish Research Agency (Agencia Estatal de Investigacion) through the Contract No. FPA201678022-P and IFT Centro de Excelencia Severo Ochoa under Grant No. SEV-2016-0597.

\section{APPENDIX: OBSERVABLES TO LO AND NLO IN THE SMEFT}

In this Appendix, we report the contributions to the observables of Table II using the definitions of Eq. (17). The contributions to the $Z$ width are,

$$
\begin{aligned}
\delta \Gamma(Z \rightarrow \nu \bar{\nu})^{\mathrm{LO}}= & \frac{v^{2}}{\Lambda^{2}}\left\{-0.3318 \mathcal{C}_{\phi l}^{(1)}+0.1659 \mathcal{C}_{l l}-0.0829 \mathcal{C}_{\phi D}\right\} \\
\delta \Gamma(Z \rightarrow \nu \bar{\nu})^{\mathrm{NLO}=}= & \frac{v^{2}}{\Lambda^{2}}\left\{-0.3446 \mathcal{C}_{\phi l}^{(1)}+0.1640 \mathcal{C}_{l l}-0.0853 \mathcal{C}_{\phi D}-0.0003 \mathcal{C}_{\phi d}-0.0003 \mathcal{C}_{\phi e}-0.0018 \mathcal{C}_{\phi l}^{(3)}-0.0073 \mathcal{C}_{\phi q}^{(1)}\right. \\
& +0.0054 \mathcal{C}_{\phi q}^{(3)}+0.0083 \mathcal{C}_{\phi u}-0.0004 \mathcal{C}_{l d}-0.0004 \mathcal{C}_{l e}-0.0061 \mathcal{C}_{l q}^{(1)}-0.0061 \mathcal{C}_{l q}^{(3)}+0.008 \mathcal{C}_{l u} \\
& \left.-0.0002 \mathcal{C}_{\phi \square}-0.0001 \mathcal{C}_{\phi W}+0.0063 \mathcal{C}_{\phi W B}+0.0001 \mathcal{C}_{u W}-0.0001 \mathcal{C}_{W}\right\} \\
\delta \Gamma\left(Z \rightarrow l^{+} l^{-}\right)^{\mathrm{LO}}= & \frac{v^{2}}{\Lambda^{2}}\left\{-0.1408 \mathcal{C}_{\phi e}+0.191 \mathcal{C}_{\phi l}^{(1)}-0.037 \mathcal{C}_{\phi l}^{(3)}+0.114 \mathcal{C}_{l l}-0.057 \mathcal{C}_{\phi D}-0.0713 \mathcal{C}_{\phi W B}\right\} \\
\delta \Gamma\left(Z \rightarrow l^{+} l^{-}\right)^{\mathrm{NLO}=}= & \frac{v^{2}}{\Lambda^{2}}\left\{-0.1597 \mathcal{C}_{\phi e}+0.1834 \mathcal{C}_{\phi l}^{(1)}-0.0221 \mathcal{C}_{\phi l}^{(3)}+0.0985 \mathcal{C}_{l l}-0.0508 \mathcal{C}_{\phi D}-0.0349 \mathcal{C}_{\phi W B}-0.0001 \mathcal{C}_{\phi W}\right. \\
& -0.0002 \mathcal{C}_{e d}-0.0005 \mathcal{C}_{e e}+0.0035 \mathcal{C}_{e u}-0.0002 \mathcal{C}_{\phi d}-0.0042 \mathcal{C}_{\phi q}^{(1)}+0.0032 \mathcal{C}_{\phi q}^{(3)}+0.0049 \mathcal{C}_{\phi u} \\
& +0.0002 \mathcal{C}_{l d}+0.0001 \mathcal{C}_{l e}+0.0034 \mathcal{C}_{l q}^{(1)}-0.0031 \mathcal{C}_{l q}^{(3)}-0.0045 \mathcal{C}_{l u}-0.0001 \mathcal{C}_{\phi \square}-0.0027 \mathcal{C}_{q e} \\
& \left.-0.0007 \mathcal{C}_{u B}-0.0007 \mathcal{C}_{u W}-0.0001 \mathcal{C}_{W}\right\}
\end{aligned}
$$




$$
\begin{aligned}
& \delta \Gamma(Z \rightarrow u \bar{u})^{\mathrm{LO}}=\delta \Gamma(Z \rightarrow c \bar{c})^{\mathrm{LO}} \\
& =\frac{v^{2}}{\Lambda^{2}}\left\{-0.9261 \mathcal{C}_{\phi l}^{(3)}-0.7138 \mathcal{C}_{\phi q}^{(1)}+0.7138 \mathcal{C}_{\phi q}^{(3)}+0.2815 \mathcal{C}_{\phi u}+0.4631 \mathcal{C}_{l l}-0.2315 \mathcal{C}_{\phi D}-0.4093 \mathcal{C}_{\phi W B}\right\} \\
& \delta \Gamma(Z \rightarrow u \bar{u})^{\mathrm{NLO}}=\delta \Gamma(Z \rightarrow c \bar{c})^{\mathrm{NLO}} \\
& =\frac{v^{2}}{\Lambda^{2}}\left\{-0.9697 \mathcal{C}_{\phi l}^{(3)}-0.7619 \mathcal{C}_{\phi q}^{(1)}+0.7537 \mathcal{C}_{\phi q}^{(3)}+0.3539 \mathcal{C}_{\phi u}+0.4673 \mathcal{C}_{l l}-0.2421 \mathcal{C}_{\phi D}-0.4049 \mathcal{C}_{\phi W B}\right. \\
& +0.0004 \mathcal{C}_{e u}-0.0013 \mathcal{C}_{\phi d}-0.0013 \mathcal{C}_{\phi e}-0.0022 \mathcal{C}_{\phi l}^{(1)}-0.0009 \mathcal{C}_{l q}^{(1)}+0.0099 \mathcal{C}_{l q}^{(3)}+0.0004 \mathcal{C}_{l u} \\
& -0.0002 \mathcal{C}_{\phi B}-0.0003 \mathcal{C}_{\phi \square}-0.0004 \mathcal{C}_{\phi W}-0.0009 \mathcal{C}_{q d}^{(1)}-0.0009 \mathcal{C}_{q e}-0.0448 \mathcal{C}_{q q}^{(1)}-0.0629 \mathcal{C}_{q q}^{(3)} \\
& \left.+0.0223 \mathcal{C}_{q u}^{(1)}-0.006 \mathcal{C}_{u B}+0.0004 \mathcal{C}_{u d}^{(1)}-0.0221 \mathcal{C}_{u u}-0.0049 \mathcal{C}_{u W}-0.0005 \mathcal{C}_{W}\right\} \\
& \delta \Gamma(Z \rightarrow d \bar{d})^{\mathrm{LO}}=\delta \Gamma(Z \rightarrow s \bar{s})^{\mathrm{LO}} \\
& =\frac{v^{2}}{\Lambda^{2}}\left\{-0.1408 \mathcal{C}_{\phi d}-1.0299 \mathcal{C}_{\phi l}^{(3)}+0.8545 \mathcal{C}_{\phi q}^{(1)}+0.8545 \mathcal{C}_{\phi q}^{(3)}+0.5149 \mathcal{C}_{l l}-0.2575 \mathcal{C}_{\phi D}-0.3379 \mathcal{C}_{\phi W B}\right\} \\
& \delta \Gamma(Z \rightarrow d \bar{d})^{\mathrm{NLO}}=\delta \Gamma(Z \rightarrow s \bar{s})^{\mathrm{NLO}} \\
& \begin{aligned}
= & \frac{v^{2}}{\Lambda^{2}}\left\{-0.1671 \mathcal{C}_{\phi d}-1.1143 \mathcal{C}_{\phi l}^{(3)}+0.8889 \mathcal{C}_{\phi q}^{(1)}+0.9222 \mathcal{C}_{\phi q}^{(3)}+0.5371 \mathcal{C}_{l l}-0.2779 \mathcal{C}_{\phi D}-0.361 \mathcal{C}_{\phi W B}\right. \\
& -0.0004 \mathcal{C}_{d d}-0.0002 \mathcal{C}_{e d}-0.0013 \mathcal{C}_{\phi e}-0.0024 \mathcal{C}_{\phi l}^{(1)}+0.0255 \mathcal{C}_{\phi u}-0.0002 \mathcal{C}_{l d}+0.0011 \mathcal{C}_{l q}^{(1)} \\
& +0.011 \mathcal{C}_{l q}^{(3)}-0.0002 \mathcal{C}_{\phi B}-0.0004 \mathcal{C}_{\phi \square}-0.0004 \mathcal{C}_{\phi W}-0.0016 \mathcal{C}_{q d}^{(1)}+0.0011 \mathcal{C}_{q e}+0.0293 \mathcal{C}_{q q}^{(1)} \\
& \left.-0.0118 \mathcal{C}_{q q}^{(3)}-0.0205 \mathcal{C}_{q u}^{(1)}-0.0053 \mathcal{C}_{u B}+0.0035 \mathcal{C}_{u d}^{(1)}-0.0041 \mathcal{C}_{u W}-0.0005 \mathcal{C}_{W}\right\}
\end{aligned} \\
& \delta \Gamma(Z \rightarrow b \bar{b})^{\mathrm{LO}}=\frac{v^{2}}{\Lambda^{2}}\left\{-0.1400 \mathcal{C}_{\phi d}-1.0242 \mathcal{C}_{\phi l}^{(3)}+0.8498 \mathcal{C}_{\phi q}^{(1)}+0.8498 \mathcal{C}_{\phi q}^{(3)}+0.5121 \mathcal{C}_{l l}-0.2561 \mathcal{C}_{\phi D}-0.3361 \mathcal{C}_{\phi W B}\right\} \\
& \delta \Gamma(Z \rightarrow b \bar{b})^{\mathrm{NLO}}=\frac{v^{2}}{\Lambda^{2}}\left\{-0.1662 \mathcal{C}_{\phi d}-1.0751 \mathcal{C}_{\phi l}^{(3)}+0.8795 \mathcal{C}_{\phi q}^{(1)}+0.8861 \mathcal{C}_{\phi q}^{(3)}+0.5177 \mathcal{C}_{l l}-0.268 \mathcal{C}_{\phi D}-0.3441 \mathcal{C}_{\phi W B}\right. \\
& -0.0004 \mathcal{C}_{d d}-0.0002 \mathcal{C}_{e d}-0.0013 \mathcal{C}_{\phi e}-0.0023 \mathcal{C}_{\phi l}^{(1)}+0.0222 \mathcal{C}_{\phi u}-0.0002 \mathcal{C}_{l d}+0.0011 \mathcal{C}_{l q}^{(1)} \\
& +0.0109 \mathcal{C}_{l q}^{(3)}-0.0002 \mathcal{C}_{\phi B}-0.0004 \mathcal{C}_{\phi \square}-0.0004 \mathcal{C}_{\phi W}-0.0016 \mathcal{C}_{q d}^{(1)}+0.0011 \mathcal{C}_{q e}+0.0292 \mathcal{C}_{q q}^{(1)} \\
& \left.-0.0117 \mathcal{C}_{q q}^{(3)}-0.0204 \mathcal{C}_{q u}^{(1)}-0.0069 \mathcal{C}_{u B}+0.0035 \mathcal{C}_{u d}^{(1)}-0.0168 \mathcal{C}_{u W}-0.0027 \mathcal{C}_{W}\right\}
\end{aligned}
$$

The SMEFT contributions to the total $Z$ width are,

$$
\begin{aligned}
\delta \Gamma_{Z}^{\mathrm{LO}}= & \frac{v^{2}}{\Lambda^{2}}\left\{-0.4223 \mathcal{C}_{\phi d}-0.4223 \mathcal{C}_{\phi e}-0.4223 \mathcal{C}_{\phi l}^{(1)}-5.053 \mathcal{C}_{\phi l}^{(3)}+1.1361 \mathcal{C}_{\phi q}^{(1)}+3.9911 \mathcal{C}_{\phi q}^{(3)}+0.5631 \mathcal{C}_{\phi u}+3.3106 \mathcal{C}_{l l}\right. \\
& \left.-1.6553 \mathcal{C}_{\phi D}-2.0463 \mathcal{C}_{\phi W B}\right\} \\
\delta \Gamma_{Z}^{\mathrm{NLO}}= & \frac{v^{2}}{\Lambda^{2}}\left\{-0.5044 \mathcal{C}_{\phi d}-0.4864 \mathcal{C}_{\phi e}-0.4948 \mathcal{C}_{\phi l}^{(1)}-5.315 \mathcal{C}_{\phi l}^{(3)}+1.0991 \mathcal{C}_{\phi q}^{(1)}+4.2636 \mathcal{C}_{\phi q}^{(3)}+0.8207 \mathcal{C}_{\phi u}+3.3141 \mathcal{C}_{l l}\right. \\
& -1.7165 \mathcal{C}_{\phi D}-1.9615 \mathcal{C}_{\phi W B}-0.0013 \mathcal{C}_{d d}-0.0011 \mathcal{C}_{e d}-0.0016 \mathcal{C}_{e e}+0.0113 \mathcal{C}_{e u}-0.0011 \mathcal{C}_{l d}-0.0011 \mathcal{C}_{l e} \\
& -0.0065 \mathcal{C}_{l q}^{(1)}+0.025 \mathcal{C}_{l q}^{(3)}+0.0113 \mathcal{C}_{l u}-0.0014 \mathcal{C}_{\phi B}-0.0028 \mathcal{C}_{\phi \square}-0.0026 \mathcal{C}_{\phi W}-0.0065 \mathcal{C}_{q d}^{(1)}-0.0065 \mathcal{C}_{q e} \\
& \left.-0.0017 \mathcal{C}_{q q}^{(1)}-0.161 \mathcal{C}_{q q}^{(3)}-0.0168 \mathcal{C}_{q u}^{(1)}-0.0318 \mathcal{C}_{u B}+0.0113 \mathcal{C}_{u d}^{(1)}-0.0443 \mathcal{C}_{u u}-0.0365 \mathcal{C}_{u W}-0.0054 \mathcal{C}_{W}\right\}
\end{aligned}
$$

The ratios are defined to be

$$
R_{l}=\frac{\Sigma_{q} \Gamma(Z \rightarrow q \bar{q})}{\Gamma(Z \rightarrow l l)} \quad R_{c}=\frac{\Gamma(Z \rightarrow u \bar{u})}{\Sigma_{q} \Gamma(Z \rightarrow q \bar{q})} \quad R_{b}=\frac{\Gamma(Z \rightarrow d \bar{d})}{\Sigma_{q} \Gamma(Z \rightarrow q \bar{q})}
$$


and the SMEFT contributions are,

$$
\begin{aligned}
& \delta R_{l}^{\mathrm{LO}}=\frac{v^{2}}{\Lambda^{2}}\left\{-4.978 \mathcal{C}_{\phi d}+33.673 \mathcal{C}_{\phi e}-45.688 \mathcal{C}_{\phi l}^{(1)}-49.393 \mathcal{C}_{\phi l}^{(3)}+13.39 \mathcal{C}_{\phi q}^{(1)}+47.041 \mathcal{C}_{\phi q}^{(3)}+6.637 \mathcal{C}_{\phi u}+1.853 \mathcal{C}_{l l}\right. \\
& \left.-0.926 \mathcal{C}_{\phi D}-4.532 \mathcal{C}_{\phi W B}\right\} \\
& \delta R_{l}^{\mathrm{NLO}}=\frac{v^{2}}{\Lambda^{2}}\left\{-5.926 \mathcal{C}_{\phi d}+39.228 \mathcal{C}_{\phi e}-45.528 \mathcal{C}_{\phi l}^{(1)}-56.848 \mathcal{C}_{\phi l}^{(3)}+14.509 \mathcal{C}_{\phi q}^{(1)}+49.697 \mathcal{C}_{\phi q}^{(3)}+8.104 \mathcal{C}_{\phi u}+5.621 \mathcal{C}_{l l}\right. \\
& -2.962 \mathcal{C}_{\phi D}-13.432 \mathcal{C}_{\phi W B}-0.015 \mathcal{C}_{d d}+0.038 \mathcal{C}_{e d}+0.124 \mathcal{C}_{e e}-0.834 \mathcal{C}_{e u}-0.063 \mathcal{C}_{l d}-0.012 \mathcal{C}_{l e}-0.795 \mathcal{C}_{l q}^{(1)} \\
& +1.362 \mathcal{C}_{l q}^{(3)}+1.083 \mathcal{C}_{l u}-0.004 \mathcal{C}_{\phi B}-0.002 \mathcal{C}_{\phi \square}-0.005 \mathcal{C}_{\phi W}-0.077 \mathcal{C}_{q d}^{(1)}+0.654 \mathcal{C}_{q e}-0.020 \mathcal{C}_{q q}^{(1)}-1.898 \mathcal{C}_{q q}^{(3)} \\
& \left.-0.198 \mathcal{C}_{q u}^{(1)}-0.168 \mathcal{C}_{u B}+0.133 \mathcal{C}_{u d}^{(1)}-0.522 \mathcal{C}_{u u}-0.254 \mathcal{C}_{u W}-0.037 \mathcal{C}_{W}\right\} \\
& \delta R_{c}^{\mathrm{LO}}=\frac{v^{2}}{\Lambda^{2}}\left\{0.0421 \mathcal{C}_{\phi d}-0.0449 \mathcal{C}_{\phi l}^{(3)}-0.5279 \mathcal{C}_{\phi q}^{(1)}+0.0164 \mathcal{C}_{\phi q}^{(3)}+0.1073 \mathcal{C}_{\phi u}+0.0224 \mathcal{C}_{l l}-0.0112 \mathcal{C}_{\phi D}-0.0549 \mathcal{C}_{\phi W B}\right\} \\
& \delta R_{c}^{\mathrm{NLO}}=\frac{v^{2}}{\Lambda^{2}}\left\{0.0490 \mathcal{C}_{\phi d}-0.0383 \mathcal{C}_{\phi l}^{(3)}-0.5499 \mathcal{C}_{\phi q}^{(1)}+0.0138 \mathcal{C}_{\phi q}^{(3)}+0.1263 \mathcal{C}_{\phi u}+0.0184 \mathcal{C}_{l l}-0.0097 \mathcal{C}_{\phi D}-0.0469 \mathcal{C}_{\phi W B}\right. \\
& +0.0001 \mathcal{C}_{d d}+0.0001 \mathcal{C}_{e d}+0.0001 \mathcal{C}_{e u}-0.0001 \mathcal{C}_{\phi e}-0.0001 \mathcal{C}_{\phi l}^{(1)}+0.0001 \mathcal{C}_{l d}-0.0007 \mathcal{C}_{l q}^{(1)}+0.0005 \mathcal{C}_{l q}^{(3)} \\
& +0.0001 \mathcal{C}_{l u}+0.0001 \mathcal{C}_{q d}^{(1)}-0.0007 \mathcal{C}_{q e}-0.0258 \mathcal{C}_{q q}^{(1)}-0.0205 \mathcal{C}_{q q}^{(3)}+0.0146 \mathcal{C}_{q u}^{(1)}-0.0005 \mathcal{C}_{u B}-0.0009 \mathcal{C}_{u d}^{(1)} \\
& \left.-0.0084 \mathcal{C}_{u u}+0.0006 \mathcal{C}_{u W}+0.0002 \mathcal{C}_{W}\right\} \\
& \delta R_{b}^{\mathrm{LO}}=\frac{v^{2}}{\Lambda^{2}}\left\{-0.02808 \mathcal{C}_{\phi d}+0.02993 \mathcal{C}_{\phi l}^{(3)}+0.3519 \mathcal{C}_{\phi q}^{(1)}-0.01094 \mathcal{C}_{\phi q}^{(3)}-0.07156 \mathcal{C}_{\phi u}-0.01497 \mathcal{C}_{l l}+0.00748 \mathcal{C}_{\phi D}\right. \\
& \left.+0.03661 \mathcal{C}_{\phi W B}\right\} \\
& \delta R_{b}^{\mathrm{NLO}}=\frac{v^{2}}{\Lambda^{2}}\left\{-0.03300 \mathcal{C}_{\phi d}+0.03298 \mathcal{C}_{\phi l}^{(3)}+0.36473 \mathcal{C}_{\phi q}^{(1)}-0.01690 \mathcal{C}_{\phi q}^{(3)}-0.08461 \mathcal{C}_{\phi u}-0.01596 \mathcal{C}_{l l}+0.00835 \mathcal{C}_{\phi D}\right. \\
& +0.03495 \mathcal{C}_{\phi W B}-0.00008 \mathcal{C}_{d d}-0.00004 \mathcal{C}_{e d}-0.00009 \mathcal{C}_{e u}+0.00007 \mathcal{C}_{\phi e}+0.00010 \mathcal{C}_{\phi l}^{(1)}-0.00004 \mathcal{C}_{l d} \\
& +0.00044 \mathcal{C}_{l q}^{(1)}-0.00034 \mathcal{C}_{l q}^{(3)}-0.00009 \mathcal{C}_{l u}+0.00001 \mathcal{C}_{\phi B}+0.00001 \mathcal{C}_{\phi W}-0.00008 \mathcal{C}_{q d}^{(1)}+0.00044 \mathcal{C}_{q e} \\
& +0.01717 \mathcal{C}_{q q}^{(1)}+0.01367 \mathcal{C}_{q q}^{(3)}-0.00972 \mathcal{C}_{q u}^{(1)}-0.00028 \mathcal{C}_{u B}+0.00060 \mathcal{C}_{u d}^{(1)}+0.00562 \mathcal{C}_{u u}-0.00534 \mathcal{C}_{u W} \\
& \left.-0.00098 \mathcal{C}_{W}\right\} \\
& \sigma_{h}^{\mathrm{LO}}=\frac{v^{2}}{\Lambda^{2}}\left\{4.05 \mathcal{C}_{\phi d}-55.524 \mathcal{C}_{\phi e}+109.235 \mathcal{C}_{\phi l}^{(1)}+32.796 \mathcal{C}_{\phi l}^{(3)}-10.896 \mathcal{C}_{\phi q}^{(1)}-38.278 \mathcal{C}_{\phi q}^{(3)}-5.4 \mathcal{C}_{\phi u}+4.319 \mathcal{C}_{l l}\right. \\
& \left.-2.16 \mathcal{C}_{\phi D}-10.565 \mathcal{C}_{\phi W B}\right\} \\
& \sigma_{h}^{\mathrm{NLO}}=\frac{v^{2}}{\Lambda^{2}}\left\{4.692 \mathcal{C}_{\phi d}-62.858 \mathcal{C}_{\phi e}+106.834 \mathcal{C}_{\phi l}^{(1)}+40.991 \mathcal{C}_{\phi l}^{(3)}-11.605 \mathcal{C}_{\phi q}^{(1)}-39.315 \mathcal{C}_{\phi q}^{(3)}-6.315 \mathcal{C}_{\phi u}-1.355 \mathcal{C}_{l l}\right. \\
& +0.829 \mathcal{C}_{\phi D}+3.311 \mathcal{C}_{\phi W B}+0.012 \mathcal{C}_{d d}-0.068 \mathcal{C}_{e d}-0.205 \mathcal{C}_{e e}+1.382 \mathcal{C}_{e u}+0.142 \mathcal{C}_{l d}+0.063 \mathcal{C}_{l e}+1.945 \mathcal{C}_{l q}^{(1)} \\
& -1.101 \mathcal{C}_{l q}^{(3)}-2.598 \mathcal{C}_{l u}-0.001 \mathcal{C}_{\phi B}+0.002 \mathcal{C}_{\phi \square}+0.063 \mathcal{C}_{q d}^{(1)}-1.064 \mathcal{C}_{q e}+0.017 \mathcal{C}_{q q}^{(1)}+1.544 \mathcal{C}_{q q}^{(3)}+0.161 \mathcal{C}_{q u}^{(1)} \\
& \left.-0.009 \mathcal{C}_{u B}-0.108 \mathcal{C}_{u d}^{(1)}+0.424 \mathcal{C}_{u u}+0.067 \mathcal{C}_{u W}+0.027 \mathcal{C}_{W}\right\}
\end{aligned}
$$

The asymmetries are defined as,

$$
A_{l}=\frac{\Gamma\left(Z \rightarrow e_{L}^{+} e_{L}^{-}\right)-\Gamma\left(Z \rightarrow e_{R}^{+} e_{R}^{-}\right)}{\Gamma\left(Z \rightarrow e^{+} e^{-}\right)} \quad A_{c}=\frac{\Gamma\left(Z \rightarrow u_{L} \bar{u}_{L}\right)-\Gamma\left(Z \rightarrow u_{R} \bar{u}_{R}\right)}{\Gamma(Z \rightarrow u \bar{u})} \quad A_{b}=\frac{\Gamma\left(Z \rightarrow d_{L} \bar{d}_{L}\right)-\Gamma\left(Z \rightarrow d_{R} \bar{d}_{R}\right)}{\Gamma(Z \rightarrow d \bar{d})}
$$

and the SMEFT contributions are, 


$$
\begin{aligned}
& \delta A_{l}^{\mathrm{LO}}=\frac{v^{2}}{\Lambda^{2}}\left\{2.1503 \mathcal{C}_{\phi e}+1.5848 \mathcal{C}_{\phi l}^{(1)}-2.7529 \mathcal{C}_{\phi l}^{(3)}+2.1689 \mathcal{C}_{l l}-1.0844 \mathcal{C}_{\phi D}-5.305 \mathcal{C}_{\phi W B}\right\} \\
& \delta A_{l}^{\mathrm{NLO}}=\frac{v^{2}}{\Lambda^{2}}\left\{+2.1666 \mathcal{C}_{\phi e}+1.8745 \mathcal{C}_{\phi l}^{(1)}-3.3587 \mathcal{C}_{\phi l}^{(3)}+2.5342 \mathcal{C}_{l l}-1.3250 \mathcal{C}_{\phi D}-6.1599 \mathcal{C}_{\phi W B}+0.0027 \mathcal{C}_{e d}+0.0076 \mathcal{C}_{e e}\right. \\
& -0.0518 \mathcal{C}_{e u}-0.0128 \mathcal{C}_{\phi d}-0.0867 \mathcal{C}_{\phi q}^{(1)}+0.0719 \mathcal{C}_{\phi q}^{(3)}+0.1190 \mathcal{C}_{\phi u}+0.0021 \mathcal{C}_{l d}+0.0049 \mathcal{C}_{l e}+0.0307 \mathcal{C}_{l q}^{(1)} \\
& +0.0098 \mathcal{C}_{l q}^{(3)}-0.0406 \mathcal{C}_{l u}-0.0026 \mathcal{C}_{\phi B}-0.0004 \mathcal{C}_{\phi \square}-0.0026 \mathcal{C}_{\phi W}+0.0392 \mathcal{C}_{q e}-0.0866 \mathcal{C}_{u B} \\
& \left.-0.0711 \mathcal{C}_{u W}-0.0046 \mathcal{C}_{W}\right\} \\
& \delta A_{c}^{\mathrm{LO}}=\frac{v^{2}}{\Lambda^{2}}\left\{-1.779 \mathcal{C}_{\phi l}^{(3)}-0.65 \mathcal{C}_{\phi q}^{(1)}+0.65 \mathcal{C}_{\phi q}^{(3)}-1.648 \mathcal{C}_{\phi u}+0.889 \mathcal{C}_{l l}-0.445 \mathcal{C}_{\phi D}-2.175 \mathcal{C}_{\phi W B}\right\} \\
& \delta A_{c}^{\mathrm{NLO}}=\frac{v^{2}}{\Lambda^{2}}\left\{-2.295 \mathcal{C}_{\phi l}^{(3)}-0.867 \mathcal{C}_{\phi q}^{(1)}+0.856 \mathcal{C}_{\phi q}^{(3)}-1.798 \mathcal{C}_{\phi u}+1.110 \mathcal{C}_{l l}-0.581 \mathcal{C}_{\phi D}-2.699 \mathcal{C}_{\phi W B}-0.002 \mathcal{C}_{e u}\right. \\
& -0.006 \mathcal{C}_{\phi d}-0.006 \mathcal{C}_{\phi e}-0.007 \mathcal{C}_{\phi l}^{(1)}-0.001 \mathcal{C}_{l q}^{(1)}+0.025 \mathcal{C}_{l q}^{(3)}-0.002 \mathcal{C}_{l u}-0.001 \mathcal{C}_{\phi B}-0.001 \mathcal{C}_{\phi W} \\
& -0.001 \mathcal{C}_{q d}^{(1)}-0.001 \mathcal{C}_{q e}-0.045 \mathcal{C}_{q q}^{(1)}-0.063 \mathcal{C}_{q q}^{(3)}-0.014 \mathcal{C}_{q u}^{(1)}-0.037 \mathcal{C}_{u B}-0.002 \mathcal{C}_{u d}^{(1)}+0.13 \mathcal{C}_{u u} \\
& \left.-0.03 \mathcal{C}_{u W}-0.002 \mathcal{C}_{W}\right\} \\
& \delta A_{b}^{\mathrm{LO}}=\frac{v^{2}}{\Lambda^{2}}\left\{0.727 \mathcal{C}_{\phi d}-0.328 \mathcal{C}_{\phi l}^{(3)}+0.12 \mathcal{C}_{\phi q}^{(1)}+0.12 \mathcal{C}_{\phi q}^{(3)}+0.164 \mathcal{C}_{l l}-0.082 \mathcal{C}_{\phi D}-0.401 \mathcal{C}_{\phi W B}\right\} \\
& \delta A_{b}^{\mathrm{NLO}}=\frac{v^{2}}{\Lambda^{2}}\left\{+0.842 \mathcal{C}_{\phi d}-0.424 \mathcal{C}_{\phi l}^{(3)}+0.149 \mathcal{C}_{\phi q}^{(1)}+0.157 \mathcal{C}_{\phi q}^{(3)}+0.205 \mathcal{C}_{l l}-0.107 \mathcal{C}_{\phi D}-0.501 \mathcal{C}_{\phi W B}+0.002 \mathcal{C}_{d d}\right. \\
& +0.001 \mathcal{C}_{e d}-0.001 \mathcal{C}_{\phi e}-0.001 \mathcal{C}_{\phi l}^{(1)}+0.009 \mathcal{C}_{\phi u}+0.001 \mathcal{C}_{l d}+0.005 \mathcal{C}_{l q}^{(3)}+0.014 \mathcal{C}_{q d}^{(1)}+0.005 \mathcal{C}_{q q}^{(1)} \\
& \left.-0.002 \mathcal{C}_{q q}^{(3)}-0.003 \mathcal{C}_{q u}^{(1)}-0.007 \mathcal{C}_{u B}-0.018 \mathcal{C}_{u d}^{(1)}-0.007 \mathcal{C}_{u W}-0.001 \mathcal{C}_{W} \cdot\right\}
\end{aligned}
$$

Finally, the forward backward asymmetries are defined as

$$
A_{F B, i}=\frac{\sigma_{F}-\sigma_{B}}{\sigma_{F}+\sigma_{B}}
$$

where defining $\theta$ to be the angle between the incoming $l^{-}$and the outgoing $\bar{f}_{i}, \sigma_{F}$ has $\theta$ between $\left(0, \frac{\pi}{2}\right)$ and $\sigma_{B}$ has $\theta$ between $\left(\frac{\pi}{2}, \pi\right)$. The SMEFT results are,

$$
\begin{aligned}
A_{F B, l}^{\mathrm{LO}}= & \frac{v^{2}}{\Lambda^{2}}\left\{0.9547 \mathcal{C}_{\phi e}+0.7037 \mathcal{C}_{\phi l}^{(1)}-1.2223 \mathcal{C}_{\phi l}^{(3)}+0.9630 \mathcal{C}_{l l}-0.4815 \mathcal{C}_{\phi D}-2.3555 \mathcal{C}_{\phi W B}\right\} \\
A_{F B, l}^{\mathrm{NLO}=} & \frac{v^{2}}{\Lambda^{2}}\left\{+0.4783 \mathcal{C}_{\phi e}+0.4138 \mathcal{C}_{\phi l}^{(1)}-0.7414 \mathcal{C}_{\phi l}^{(3)}+0.5594 \mathcal{C}_{l l}-0.2925 \mathcal{C}_{\phi D}-1.3598 \mathcal{C}_{\phi W B}+0.0006 \mathcal{C}_{e d}+0.0017 \mathcal{C}_{e e}\right. \\
& -0.0114 \mathcal{C}_{e u}-0.0028 \mathcal{C}_{\phi d}-0.0191 \mathcal{C}_{\phi q}^{(1)}+0.0159 \mathcal{C}_{\phi q}^{(3)}+0.0263 \mathcal{C}_{\phi u}+0.0005 \mathcal{C}_{l d}+0.0011 \mathcal{C}_{l e}+0.0068 \mathcal{C}_{l q}^{(1)} \\
& +0.0022 \mathcal{C}_{l q}^{(3)}-0.009 \mathcal{C}_{l u}-0.0006 \mathcal{C}_{\phi B}-0.0001 \mathcal{C}_{\phi \square}-0.0006 \mathcal{C}_{\phi W}+0.0086 \mathcal{C}_{q e}-0.0191 \mathcal{C}_{u B}-0.0157 \mathcal{C}_{u W} \\
& \left.-0.0010 \mathcal{C}_{W}\right\} \\
A_{F B, c}^{\mathrm{LO}}= & \frac{v^{2}}{\Lambda^{2}}\left\{1.1785 \mathcal{C}_{\phi e}+0.8686 \mathcal{C}_{\phi l}^{(1)}-1.9036 \mathcal{C}_{\phi l}^{(3)}-0.1443 \mathcal{C}_{\phi q}^{(1)}+0.1443 \mathcal{C}_{\phi q}^{(3)}-0.3658 \mathcal{C}_{\phi u}+1.3861 \mathcal{C}_{l l}-0.693 \mathcal{C}_{\phi D}\right. \\
& \left.-3.3903 \mathcal{C}_{\phi W B}\right\}
\end{aligned}
$$




$$
\begin{aligned}
A_{F B, c}^{\mathrm{NLO}}= & \frac{v^{2}}{\Lambda^{2}}\left\{+1.0846 \mathcal{C}_{\phi e}+0.9381 \mathcal{C}_{\phi l}^{(1)}-1.9356 \mathcal{C}_{\phi l}^{(3)}-0.1391 \mathcal{C}_{\phi q}^{(1)}+0.1305 \mathcal{C}_{\phi q}^{(3)}-0.1388 \mathcal{C}_{\phi u}+1.3918 \mathcal{C}_{l l}-0.7278 \mathcal{C}_{\phi D}\right. \\
& -3.3833 \mathcal{C}_{\phi W B}+0.0014 \mathcal{C}_{e d}+0.0038 \mathcal{C}_{e e}-0.0262 \mathcal{C}_{e u}-0.007 \mathcal{C}_{\phi d}+0.0011 \mathcal{C}_{l d}+0.0024 \mathcal{C}_{l e}+0.0153 \mathcal{C}_{l q}^{(1)} \\
& +0.0076 \mathcal{C}_{l q}^{(3)}-0.0206 \mathcal{C}_{l u}-0.0014 \mathcal{C}_{\phi B}-0.0002 \mathcal{C}_{\phi \square}-0.0014 \mathcal{C}_{\phi W}-0.0001 \mathcal{C}_{q d}^{(1)}+0.0195 \mathcal{C}_{q e}-0.0050 \mathcal{C}_{q q}^{(1)} \\
& \left.-0.0070 \mathcal{C}_{q q}^{(3)}-0.0016 \mathcal{C}_{q u}^{(1)}-0.0475 \mathcal{C}_{u B}-0.0002 \mathcal{C}_{u d}^{(1)}+0.0143 \mathcal{C}_{u u}-0.0389 \mathcal{C}_{u W}-0.0025 \mathcal{C}_{W}\right\} \\
A_{F B, b}^{\mathrm{LO}}= & \frac{v^{2}}{\Lambda^{2}}\left\{0.1615 \mathcal{C}_{\phi d}+1.5275 \mathcal{C}_{\phi e}+1.1258 \mathcal{C}_{\phi l}^{(1)}-2.0284 \mathcal{C}_{\phi l}^{(3)}+0.0266 \mathcal{C}_{\phi q}^{(1)}+0.0266 \mathcal{C}_{\phi q}^{(3)}+1.5771 \mathcal{C}_{l l}-0.7886 \mathcal{C}_{\phi D}\right. \\
& \left.-3.8576 \mathcal{C}_{\phi W B}\right\} \\
A_{F B, b}^{\mathrm{NLO}}= & \frac{v^{2}}{\Lambda^{2}}\left\{+0.0840 \mathcal{C}_{\phi d}+1.5062 \mathcal{C}_{\phi e}+1.3031 \mathcal{C}_{\phi l}^{(1)}-2.3819 \mathcal{C}_{\phi l}^{(3)}-0.0439 \mathcal{C}_{\phi q}^{(1)}+0.0673 \mathcal{C}_{\phi q}^{(3)}+1.7845 \mathcal{C}_{l l}-0.9331 \mathcal{C}_{\phi D}\right. \\
& -4.3379 \mathcal{C}_{\phi W B}+0.0002 \mathcal{C}_{d d}+0.0020 \mathcal{C}_{e d}+0.0053 \mathcal{C}_{e e}-0.0360 \mathcal{C}_{e u}+0.0838 \mathcal{C}_{\phi u}+0.0016 \mathcal{C}_{l d}+0.0034 \mathcal{C}_{l e} \\
& +0.0214 \mathcal{C}_{l q}^{(1)}+0.0073 \mathcal{C}_{l q}^{(3)}-0.0283 \mathcal{C}_{l u}-0.0018 \mathcal{C}_{\phi B}-0.0003 \mathcal{C}_{\phi \square}-0.0018 \mathcal{C}_{\phi W}+0.0015 \mathcal{C}_{q d}^{(1)}+0.0273 \mathcal{C}_{q e} \\
& \left.+0.0005 \mathcal{C}_{q q}^{(1)}-0.0002 \mathcal{C}_{q q}^{(3)}-0.0003 \mathcal{C}_{q u}^{(1)}-0.0610 \mathcal{C}_{u B}-0.0020 \mathcal{C}_{u d}^{(1)}-0.0502 \mathcal{C}_{u W}-0.0033 \mathcal{C}_{W}\right\} .
\end{aligned}
$$

[1] S. Dawson, C. Englert, and T. Plehn, Higgs physics: It ain't over till it's over, Phys. Rep. 816, 1 (2019).

[2] I. Brivio and M. Trott, The Standard Model as an effective field theory, Phys. Rep. 793, 1 (2019).

[3] A. Biekotter, T. Corbett, and T. Plehn, The gauge-Higgs legacy of the LHC run II, SciPost Phys. 6, 064 (2019).

[4] E. da Silva Almeida, A. Alves, N. Rosa Agostinho, O. J. P. Eboli, and M. C. Gonzalez-Garcia, Electroweak sector under scrutiny: A combined analysis of LHC and electroweak precision data, Phys. Rev. D 99, 033001 (2019).

[5] J. de Blas, M. Ciuchini, E. Franco, S. Mishima, M. Pierini, L. Reina, and L. Silvestrini, The global electroweak and Higgs fits in the LHC era, Proc. Sci. EPS-HEP2017 (2017) 467 [arXiv:1710.05402].

[6] S. Di Vita, C. Grojean, G. Panico, M. Riembau, and T. Vantalon, A global view on the Higgs self-coupling, J. High Energy Phys. 09 (2017) 069.

[7] J. Ellis, C. W. Murphy, V. Sanz, and T. You, Updated global SMEFT fit to Higgs, Diboson and electroweak data, J. High Energy Phys. 06 (2018) 146.

[8] C. Grojean, M. Montull, and M. Riembau, Diboson at the LHC vs LEP, J. High Energy Phys. 03 (2019) 020.

[9] L. Berthier, M. Bjorn, and M. Trott, Incorporating doubly resonant $W^{ \pm}$data in a global fit of SMEFT parameters to lift flat directions, J. High Energy Phys. 09 (2016) 157.

[10] A. Pomarol and F. Riva, Towards the ultimate SM fit to close in on Higgs physics, J. High Energy Phys. 01 (2014) 151.

[11] J. Baglio, S. Dawson, and I. M. Lewis, An NLO QCD effective field theory analysis of $W^{+} W^{-}$production at the LHC including fermionic operators, Phys. Rev. D 96, 073003 (2017).

[12] J. Baglio, S. Dawson, and I. M. Lewis, NLO effects in EFT fits to $W^{+} W^{-}$production at the LHC, Phys. Rev. D 99, 035029 (2019).
[13] N. P. Hartland, F. Maltoni, E. R. Nocera, J. Rojo, E. Slade, E. Vryonidou, and C. Zhang, A Monte Carlo global analysis of the Standard Model effective field theory: The top quark sector, J. High Energy Phys. 04 (2019) 100.

[14] D. Buarque Franzosi, E. Vryonidou, and C. Zhang, Scalar production and decay to top quarks including interference effects at NLO in QCD in an EFT approach, J. High Energy Phys. 10 (2017) 096.

[15] G. Durieux, J. Gu, E. Vryonidou, and C. Zhang, Probing top-quark couplings indirectly at Higgs factories, Chin. Phys. C 42, 123107 (2018).

[16] R. Boughezal, C.-Y. Chen, F. Petriello, and D. Wiegand, Top quark decay at next-to-leading order in the Standard Model effective field theory, Phys. Rev. D 100, 056023 (2019).

[17] J. M. Cullen, B. D. Pecjak, and D. J. Scott, NLO corrections to $h \rightarrow b \bar{b}$ decay in SMEFT, J. High Energy Phys. 08 (2019) 173.

[18] R. Gauld, B. D. Pecjak, and D. J. Scott, QCD radiative corrections for $h \rightarrow b \bar{b}$ in the Standard Model dimension-6 EFT, Phys. Rev. D 94, 074045 (2016).

[19] R. Gauld, B. D. Pecjak, and D. J. Scott, One-loop corrections to $h \rightarrow b \bar{b}$ and $h \rightarrow \tau \bar{\tau}$ decays in the Standard Model dimension-6 EFT: Four-fermion operators and the large- $m_{t}$ limit, J. High Energy Phys. 05 (2016) 080.

[20] S. Dawson and P. P. Giardino, Electroweak corrections to Higgs boson decays to $\gamma \gamma$ and $W^{+} W^{-}$in standard model EFT, Phys. Rev. D 98, 095005 (2018).

[21] A. Dedes, M. Paraskevas, J. Rosiek, K. Suxho, and L. Trifyllis, The decay $h \rightarrow \gamma \gamma$ in the Standard-Model effective field theory, J. High Energy Phys. 08 (2018) 103.

[22] C. Hartmann and M. Trott, Higgs Decay to Two Photons at One Loop in the Standard Model Effective Field Theory, Phys. Rev. Lett. 115, 191801 (2015). 
[23] C. Hartmann and M. Trott, On one-loop corrections in the standard model effective field theory: The $\Gamma(h \rightarrow \gamma \gamma)$ case, J. High Energy Phys. 07 (2015) 151.

[24] S. Dawson and P. P. Giardino, Higgs decays to $Z Z$ and $Z \gamma$ in the Standard Model effective field theory: An NLO analysis, Phys. Rev. D 97, 093003 (2018).

[25] A. Dedes, K. Suxho, and L. Trifyllis, The decay $h \rightarrow Z \gamma$ in the Standard-Model effective field theory, J. High Energy Phys. 06 (2019) 115.

[26] S. Dawson, P. P. Giardino, and A. Ismail, Standard Model EFT and the Drell-Yan process at high energy, Phys. Rev. D 99, 035044 (2019).

[27] S. Dawson and A. Ismail, Standard Model EFT corrections to Z boson decays, Phys. Rev. D 98, 093003 (2018).

[28] M. Trott, EWPD in the SMEFT and the $\mathcal{O}\left(y_{t}^{2}, \lambda\right)$ one loop $Z$ decay width, in Proceedings, 52nd Rencontres de Moriond on Electroweak Interactions and Unified Theories: La Thuile, Italy (2017), pp. 63-70.

[29] C. Hartmann, W. Shepherd, and M. Trott, The $Z$ decay width in the SMEFT: $y_{t}$ and $\lambda$ corrections at one loop, J. High Energy Phys. 03 (2017) 060.

[30] W. Buchmuller and D. Wyler, Effective Lagrangian analysis of new interactions and flavor conservation, Nucl. Phys. B268, 621 (1986).

[31] B. Grzadkowski, M. Iskrzynski, M. Misiak, and J. Rosiek, Dimension-six terms in the Standard Model Lagrangian, J. High Energy Phys. 10 (2010) 085.

[32] A. Dedes, W. Materkowska, M. Paraskevas, J. Rosiek, and K. Suxho, Feynman rules for the Standard Model effective field theory in $R_{\xi}$-gauges, J. High Energy Phys. 06 (2017) 143.

[33] R. Alonso, E. E. Jenkins, A. V. Manohar, and M. Trott, Renormalization group evolution of the Standard Model dimension six operators III: Gauge coupling dependence and phenomenology, J. High Energy Phys. 04 (2014) 159.

[34] L. Berthier and M. Trott, Towards consistent electroweak precision data constraints in the SMEFT, J. High Energy Phys. 05 (2015) 024.

[35] W. F. L. Hollik, Radiative corrections in the Standard Model and their role for precision tests of the electroweak theory, Fortschr. Phys. 38, 165 (1990).

[36] I. Dubovyk, A. Freitas, J. Gluza, T. Riemann, and J. Usovitsch, Electroweak pseudo-observables and Z-boson form factors at two-loop accuracy, J. High Energy Phys. 08 (2019) 113.

[37] E. E. Jenkins, A. V. Manohar, and M. Trott, Renormalization group evolution of the Standard Model dimension six operators I: Formalism and lambda dependence, J. High Energy Phys. 10 (2013) 087.

[38] E. E. Jenkins, A. V. Manohar, and M. Trott, Renormalization group evolution of the Standard Model dimension six operators II: Yukawa dependence, J. High Energy Phys. 01 (2014) 035.

[39] C.-Y. Chen, S. Dawson, and C. Zhang, Electroweak effective operators and Higgs physics, Phys. Rev. D 89, 015016 (2014).

[40] M. Ghezzi, R. Gomez-Ambrosio, G. Passarino, and S. Uccirati, NLO Higgs effective field theory and $\kappa$-framework, J. High Energy Phys. 07 (2015) 175.

[41] T. Hahn, Generating Feynman diagrams and amplitudes with FeynArts 3, Comput. Phys. Commun. 140, 418 (2001).
[42] A. Alloul, N. D. Christensen, C. Degrande, C. Duhr, and B. Fuks, FeynRules 2.0-A complete toolbox for tree-level phenomenology, Comput. Phys. Commun. 185, 2250 (2014).

[43] R. Mertig, M. Bohm, and A. Denner, FEYN CALC: Computer algebraic calculation of Feynman amplitudes, Comput. Phys. Commun. 64, 345 (1991).

[44] V. Shtabovenko, R. Mertig, and F. Orellana, New developments in FeynCalc 9.0, Comput. Phys. Commun. 207, 432 (2016).

[45] T. Hahn, Automatic loop calculations with FeynArts, FormCalc, and LoopTools, Nucl. Phys. B, Proc. Suppl. 89, 231 (2000).

[46] C. Anastasiou and K. Melnikov, Higgs boson production at hadron colliders in NNLO QCD, Nucl. Phys. B646, 220 (2002).

[47] O. V. Tarasov, Generalized recurrence relations for two loop propagator integrals with arbitrary masses, Nucl. Phys. B502, 455 (1997).

[48] S. P. Martin, Evaluation of two loop self-energy basis integrals using differential equations, Phys. Rev. D 68, 075002 (2003).

[49] A. V. Smirnov, FIRE5: A C++ implementation of Feynman Integral REduction, Comput. Phys. Commun. 189, 182 (2015).

[50] Particle Data Group, Review of particle physics, Phys. Rev. D 98, 030001 (2018).

[51] A. Freitas, Higher-order electroweak corrections to the partial widths and branching ratios of the $\mathrm{Z}$ boson, J. High Energy Phys. 04 (2014) 070.

[52] I. Dubovyk, A. Freitas, J. Gluza, T. Riemann, and J. Usovitsch, Complete electroweak two-loop corrections to $\mathrm{Z}$ boson production and decay, Phys. Lett. B 783, 86 (2018).

[53] S. Schael et al. (ALEPH, DELPHI, L3, OPAL, SLD, LEP Electroweak Working Group, SLD Electroweak Group, SLD Heavy Flavour Group Collaborations), Precision electroweak measurements on the $Z$ resonance, Phys. Rep. 427, 257 (2006).

[54] M. Awramik, M. Czakon, and A. Freitas, Electroweak twoloop corrections to the effective weak mixing angle, J. High Energy Phys. 11 (2006) 048.

[55] M. Awramik, M. Czakon, A. Freitas, and B. A. Kniehl, Two-loop electroweak fermionic corrections to $\sin ^{2} \times \theta_{e f f}^{b \bar{b}}$, Nucl. Phys. B813, 174 (2009).

[56] T. T. E. W. Group (CDF, D0 Collaboration), Combination of the CDF and DO Effective Leptonic Electroweak Mixing Angles (2016), http://lss.fnal.gov/archive/2016/conf/ fermilab-conf-16-295-e.pdf.

[57] M. Awramik, M. Czakon, A. Freitas, and G. Weiglein, Precise prediction for the $\mathrm{W}$ boson mass in the Standard Model, Phys. Rev. D 69, 053006 (2004).

[58] J. Erler and M. Schott, Electroweak precision tests of the Standard Model after the discovery of the Higgs boson, Prog. Part. Nucl. Phys. 106, 68 (2019).

[59] G.-C. Cho, K. Hagiwara, Y. Matsumoto, and D. Nomura, The MSSM confronts the precision electroweak data and the muon g-2, J. High Energy Phys. 11 (2011) 068.

[60] L. Berthier and M. Trott, Consistent constraints on the Standard Model effective field theory, J. High Energy Phys. 02 (2016) 069. 
[61] T. Corbett, O. J. P. Eboli, and M. C. Gonzalez-Garcia, Unitarity constraints on dimension-six operators II: Including Fermionic operators, Phys. Rev. D 96, 035006 (2017).

[62] A. Falkowski and F. Riva, Model-independent precision constraints on dimension-6 operators, J. High Energy Phys. 02 (2015) 039.

[63] T. Corbett, O. J. P. Eboli, J. Gonzalez-Fraile, and M. C. Gonzalez-Garcia, Robust determination of the Higgs couplings: Power to the data, Phys. Rev. D 87, 015022 (2013).

[64] J. Elias-Miro, J. R. Espinosa, E. Masso, and A. Pomarol, Higgs windows to new physics through $d=6$ operators: Constraints and one-loop anomalous dimensions, J. High Energy Phys. 11 (2013) 066.

[65] See Supplemental Material at http://link.aps.org/supplemental/ 10.1103/PhysRevD.101.013001 which contains text expressions for the LO and NLO observables described in the text. 